\title{
A Dynamical Adaptive Resonance Architecture
}

\author{
Gregory L. Heileman, Member, IEEE, Michael Georgiopoulos, Member, IEEE, and Chaouki Abdallah, Member, IEEE
}

\begin{abstract}
A set of nonlinear differential equations that describe the dynamics of the ART1 model are presented, along with the motivation for their use. These equations are extensions of those developed by Carpenter and Grossberg [1]. It is shown how these differential equations allow the ART1 model to be realized as a collective nonlinear dynamical system. Specifically, we present an ART1-based neural network model whose description requires no external control features. That is, the dynamics of the model are completely determined by the set of coupled differential equations that comprise the model. It is shown analytically how the parameters of this model can be selected so as to guarantee a behavior equivalent to that of ART1 in both fast and slow learning scenarios. Simulations are performed in which the trajectories of node and weight activities are determined using numerical approximation techniques.
\end{abstract}

\section{INTRODUCTION}

$\mathbf{T}$ HE ART1 neural network model is a self-organizing architecture capable of learning recognition categories of complex binary input patterns. The behavior of the ART1 network is effectively described in [1, sections 3-6]. Furthermore, many of the features of the ART1 model are specified via a set of nonlinear differential equations [1, section 12]. It should be noted that a number of mechanisms in the original ART1 model-such as the reset mechanism, and the resetting of node activities to zero prior to a pattern presentation-are only qualitatively described in [1]. The focus of our work is to provide a nonlinear dynamical system model that completely captures all aspects of the behavior of the ART1 network. For the sake of convenience we will refer to the dynamical system model presented here as the augmented ART1 network (AART1-NN), as opposed to the ART1 network (ART1-NN) presented in [1]. ${ }^{1}$

There are a number of advantages offered by the dynamical system model described here. First, it is intuitively pleasing to provide a complete mathematical description of the ART1 model. After all, this model is more than just a pattern clustering technique-it is a neural network architecture, with appropriate interconnections and describing equations, which as a whole exhibits pattern clustering capabilities. Second, the analysis of dynamical systems is a well understood and rich

Manuscript received September 24, 1993; revised March 5, 1993. This work was supported in part by the Boeing Computer Services under Contract W-300445, and the Florida High Technology and Industry Council.

G. L. Heileman and C. Abdallah are with the ICS Group in the Department of Electrical and Computer Engineering, University of New Mexico, Albuquerque, NM 87131 USA.

M. Georgiopoulos is with the Department of Electrical and Computer Engineering, University of Central Florida, Orlando, FL 32816 USA.

IEEE Log Number 9208989.

${ }^{1}$ In Hirsch's classification scheme [2], the AART1-NN is classified as a dynamical system in the Cartesian product of the weight space and the node activation space. This is due to the fact that the weights in this network are adapted concurrently with the activation dynamics of the nodes. area as witnessed by the recent growth in the sciences of chaos and nonlinear physics [3], [4]. A dynamical system setting, where a neural network is allowed to follow a trajectory set by the initial conditions and the external inputs, is a natural medium for studying the stability, structure, and capabilities of a network [5]. Moreover, such a setting allows us to generalize a particular network structure (e.g., the ART1 model) in order to obtain those generic properties satisfied by the network. For example, using a dynamical system setting, Kosko was able to prove the general BAM theorems [5], and we were able to use the concept of gradient systems [6] in order to generalize the structure and update rules of an oncenter-off-surround network that is a simplified version of the dynamical system presented here [7]. Finally, a complete dynamical system description of the ART1 model facilitates its implementation in hardware. A circuit that implements the system of equations describing the ART1 model presented here utilizing analog electronic components has been successfully designed and verified using the PSpice circuit simulator [8], [9].

To put this dynamical system model of ART1 into perspective, it is useful to consider some related work. There has been much interest in reformulating the popular backpropagation algorithm using the dynamical system framework discussed above. For example, Pineda presented a backpropagation technique for exploiting the dynamics of a general class of neurodynamical systems [10], Williams proposed a learning algorithm for a continually running fully recurrent network [11], and Narendra and Parthasarathy discussed dynamic back-propagation as applied to recurrent networks [12]. In the area of adaptive resonance networks, the ART2 network [13] — which is used to classify analog input patterns - has been extended so as to allow a complete description of the model as a dynamical system [14].

The organization of the paper is as follows. In Section II descriptions of the architecture, network equations, and operation of the ART1 neural network (ART1-NN) are provided. This review leads to a presentation in Section III of the augmented ART1 neural network (AART1-NN). Section IV demonstrates that the AART1-NN equations exhibit a behavior identical to the ART1-NN behavior described in [1, sections 3-6]. This identical behavior is established under the assumption that the AART1-NN parameter values satisfy certain constraints. These constraints are also derived in Section IV. In Section V AART1-NN parameter values are chosen for an example network so as to satisfy the parameter constraints developed in Section IV. In Section VI we present computer simulation results that demonstrate the behavior of the AART1-NN for a number of different scenarios. Section VII summarizes our results and presents some concluding remarks. 


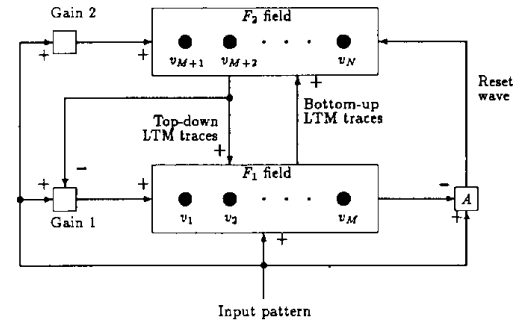

Fig. 1. The architecture of the ART1 neural network model.

\section{THE ART1 NeURAL NETWORK}

In the following sections we briefly summarize the ART1NN architecture, operation, and equations that describe the network dynamics. A more complete description of the ART1 model is given in [1], and a number of useful results and theorems regarding the capabilities of this model are given in $[1],[15],[16]$.

\section{A. Architecture}

The major components of the ART1 model are depicted in Fig. 1. These components can be grouped into two subsystems - the attentional and orienting subsystems. The $F_{1}$ and $F_{2}$ fields in the attentional subsystem each consist of a single layer of nodes. These nodes are used to encode patterns of short term memory (STM) activity, while the weighted connections between the nodes in the $F_{1}$ and $F_{2}$ fields are used to store long term memory (LTM) traces. Each node in the $F_{1}$ field is connected via bottom-up connections to all nodes in the $F_{2}$ field, and each node in the $F_{2}$ field is connected via top-down connections to each of the $F_{1}$ field nodes. In addition, the set of nodes comprising the $F_{2}$ field are completely connected.

The orienting subsystem, $A$, receives input from the $F_{1}$ field nodes, as well as from the input pattern. The orienting subsystem will generate a reset wave to the $F_{2}$ field whenever the input pattern is not matched close enough to the pattern of STM activity across the $F_{1}$ field.

\section{B. Operation}

The operation of the ART1-NN can be described as follows. STM activity is induced in the $F_{1}$ field by the introduction of an input pattern. The components of the input pattern comprise the bottom-up input to the $F_{1}$ field. A node with activity below or above its threshold is said to be subliminally or supraliminally active, respectively. The threshold is typically a small positive constant. A node is said to be activated if its activity increases from a level below its threshold to a level above its quenching threshold. In addition, a node is said to be deactivated if its activity drops from a level above its threshold to a level below its threshold. The orienting subsystem, $A$, is nonspecifically activated by the input pattern. The STM activity across the $F_{1}$ field generates an output from the $F_{1}$ field that inhibits $A$. This output activity is multiplied by the bottom-up LTM traces, and the result is a bottomup input which is supplied to the $F_{2}$ field. Next, a contrast enhancement process (competition cycle) occurs among the
$F_{2}$ field nodes, generating a STM activity across the $F_{2}$ field. A special case of this contrast enhancement mechanism is one in which only one node is chosen to remain supraliminally active in the $F_{2}$ field. This form of contrast enhancement, often referred to as a gated dipole field, is assumed throughout this paper. The output activity of the $F_{2}$ field is transformed through a multiplication process with the top-down LTM traces to generate a top-down input to the $F_{1}$ field. At this point, new STM activity is generated across the $F_{1}$ field. If there is significant mismatch between bottom-up and top-down inputs at the $F_{1}$ field, this new STM activity results in a new output activity from the $F_{1}$ field which causes a decrease in the total inhibition impinging upon $A$ from the $F_{1}$ field. As a result, the input-driven activation of $A$ may release a nonspecific reset wave which inhibits the STM activity at the $F_{2}$ field. This inhibition leads to the elimination of the top-down input affecting the activity of the $F_{1}$ field nodes. Hence, the initial STM activity is reinstated across the $F_{1}$ field. Once again, this STM activity across the $F_{1}$ field generates an output from the $F_{1}$ field which produces the same bottom-up input at the $F_{2}$ field as before. Since the node initially chosen in the $F_{2}$ field remains inhibited, a new node in the $F_{2}$ field can now be chosen. If once more the new top-down input significantly mismatches the bottom-up input at the $F_{1}$ field, then the search for an appropriate $F_{2}$ field node continues until a node is found that does not lead to a reset, or until all nodes in the $F_{2}$ field are found inappropriate to code (i.e., learn) the input pattern. If a reset wave is not generated by the orienting system after the activation of an $F_{2}$ field node, then this node is said to code the input pattern.

\section{Network Equations}

The operation of the ART1 network discussed above can be represented by a set of nonlinear differential equations. The activity of the network nodes is described by the following differential equation:

$$
\epsilon \frac{d}{d t} x=-x+(1-A x) J^{+}-(B+C x) J^{-}
$$

where $x$ is the nodal activity; while $J^{+}$and $J^{-}$, which represent the total excitatory and inhibitory input to the node, respectively, are functions of $x$. Equation (1) is called a shunting differential equation because $J^{+}$and $J^{-}$multiply the node activity $x$. Note that if $A>0$ and $C>0$, then the activity of the node remains in the bounded range $\left[-B C^{-1}, A^{-1}\right]$ no matter how large $J^{+}$and $J^{-}$become, assuming the node activity is initially in this range. Also notice that the activity of the node decays to a resting level of 0 when $J^{+}=J^{-}=0$.

We denote nodes in the $F_{1}$ field by $v_{i}$ and nodes in the $F_{2}$ field by $v_{j}$. The index of the nodes in the $F_{1}$ field ranges from 1 to $M$, while the index of the nodes in the $F_{2}$ field ranges from $M+1$ to $N$. We also denote the activity of a node $v_{i}$ by $x_{i}$, and the activity of a node $v_{j}$ by $x_{j}$. In particular, the activity of a node $v_{i}$ in the $F_{1}$ field satisfies the following differential equation

$$
\epsilon_{1} \frac{d}{d t} x_{i}=-x_{i}+\left(1-A_{1} x_{i}\right) J_{i}^{+}-\left(B_{1}+C_{1} x_{i}\right) J_{i}^{-}
$$


The total excitatory input to node $v_{i}$ is given by

$$
J_{i}^{+}=I_{i}+D_{1} \sum_{j} f_{2}\left(x_{j}\right) z_{j i}
$$

where $D_{1}$ is a constant, $I_{i}$ is the component of the binary input pattern $I$ that is received by node $v_{i}, f_{2}\left(x_{j}\right)$ is the output activity generated by node $v_{j}$ with activity $x_{j}$, and $z_{j i}$ is the value of the top-down LTM trace corresponding to the connection between node $v_{j}$ in the $F_{2}$ field and node $v_{i}$ in the $F_{1}$ field. In (3), and throughout this paper, we will assume that the output activity generated by a node $v_{j}$ with activity $x_{j}$ is the threshold function

$$
f_{2}\left(x_{j}\right)= \begin{cases}1, & \text { if } x_{j}>\delta_{2} \\ 0, & \text { otherwise }\end{cases}
$$

where $\delta_{2}$ is the threshold of every node $v_{j}$ in the $F_{2}$ field. The total inhibitory input to node $v_{i}$ is given by

$$
J_{i}^{-}=\sum_{j} f_{2}\left(x_{j}\right)
$$

The activity of a node $v_{j}$ in the $F_{2}$ field satisfies the following differential equation

$$
\epsilon_{2} \frac{d}{d t} x_{j}=-x_{j}+\left(1-A_{2} x_{j}\right) J_{j}^{+}-\left(B_{2}+C_{2} x_{j}\right) J_{j}^{-} .
$$

The total excitatory input to node $v_{j}$ is calculated as

$$
J_{j}^{+}=f_{2}\left(x_{j}\right)+T_{j}
$$

with

$$
T_{j}=D_{2} \sum_{i} f_{1}\left(x_{i}\right) z_{i j}
$$

where $D_{2}$ is a constant. In (8), and throughout this paper, we will assume that the output activity generated by a node $v_{i}$ with activity $x_{i}$ is the threshold function

$$
f_{1}\left(x_{i}\right)= \begin{cases}1, & \text { if } x_{i}>\delta_{1} \\ 0, & \text { otherwise }\end{cases}
$$

where $\delta_{1}$ is the threshold of every node $v_{i}$ in the $F_{1}$ field Finally, the total inhibitory input to a node $v_{j}$ in the $F_{2}$ field is given by

$$
J_{j}^{-}=\sum_{k \neq j} f_{2}\left(x_{k}\right)
$$

The value of the bottom-up LTM trace, $z_{i j}$, associated with an arc connecting node $v_{i}$ in the $F_{1}$ field to node $v_{j}$ in the $F_{2}$ field is determined by the following differential equation

$$
\epsilon_{z} \frac{d}{d t} z_{i j}=K_{1} f_{2}\left(x_{j}\right)\left[-E_{i j} z_{i j}+f_{1}\left(x_{i}\right)\right] \text {. }
$$

In the present model, $K_{1}$ is a constant and $E_{i j}$ is given by

$$
E_{i j}=f_{1}\left(x_{i}\right)+L^{-1} \sum_{k \neq i} f_{1}\left(x_{k}\right)
$$

where $L$ is a constant $>1$. Combining (11) and (12) yields

$$
\begin{array}{r}
\epsilon_{z} \frac{d}{d t} z_{i j}=-K\left[(L-1) f_{1}\left(x_{i}\right)+\sum_{k} f_{1}\left(x_{k}\right)\right] \\
\cdot f_{2}\left(x_{j}\right) z_{i j}+K L f_{1}\left(x_{i}\right) f_{2}\left(x_{j}\right)
\end{array}
$$

with $K=K_{1} L^{-1}$.
The value of the top-down LTM trace, $z_{j i}$, associated with an arc connecting node $v_{j}$ in the $F_{2}$ field to node $v_{i}$ in the $F_{1}$ field is determined by the following differential equation:

$$
\epsilon_{z} \frac{d}{d t} z_{j i}=-K_{2} E_{j i} f_{2}\left(x_{j}\right) z_{j i}+K_{2} f_{1}\left(x_{i}\right) f_{2}\left(x_{j}\right) .
$$

The present model assumes that

$$
K_{2}=E_{j i}=1 .
$$

The parameters $\epsilon_{1}, \epsilon_{2}$, and $\epsilon_{z}$ that appear in the previous equations are referred to as learning rates. These values determine the rate of change of the variables (STM activity in the $F_{1}$ field, STM activity in the $F_{2}$ field, and bottom-up or top-down LTM traces between pairs of nodes in the $F_{1}$ and $F_{2}$ fields) that are characterized by the above equations. A smaller value for the parameter $\epsilon$ results in a faster rate of change of the variable described by the differential equation under consideration. For example, if $\epsilon_{1}, \epsilon_{2}<<\epsilon_{z}$, then the rate of change of the STM activity in the $F_{1}$ and $F_{2}$ fields is much faster than the rate of change of the bottom-up and top-down LTM traces between pairs of nodes in the $F_{1}$ and $F_{2}$ fields.

\section{The Augmented ART1 Neural Network}

A number of implementation issues that are not directly addressed in the ART1 model [1] are considered here. These include:

i) The manner in which the mismatch-mediated reset wave can be generated.

ii) The approach taken to ensure that an $F_{2}$ field node remains inactive, once it is reset, until a new input pattern is presented.

iii) A way of automatically driving the activity of every node in the network to its resting value of zero whenever an input pattern is removed from the network.

Below we address each of these issues separately. The approach taken to resolve the aforementioned issues is directed towards a solution that will facilitate a dynamical system realization of the ART1-NN. The resolution of these issues will involve the addition of nodes in the ART1-NN architecture, and minor modifications to the original ART1 neural network equations presented in Section II-C. The resulting model is termed the AART1-NN. The major components of the AART1-NN are shown in Fig. 2. It is instructive to compare the ART1-NN architecture of Fig. 2 to the AART1NN architecture depicted in Fig. 1. One immediate observation is that the $F_{2}$ field nodes in the ART1-NN correspond to the first layer of nodes in the $F_{2}$ field of the AART1-NN architecture. That is, the first layer of nodes in the $F_{2}$ field of the AART1-NN is used for category representation, as is the $F_{2}$ field in the ART1-NN.

Resolution of Issue $i$ : Let $|I|$ denote the number of input pathways which receive positive input when the input pattern $I$ is presented. Also, let $|X|$ denote the number of nodes in the $F_{1}$ field that are supraliminally active during the presentation of the input pattern $I$. In the ART1 model, each of the $|I|$ input pathways sends an excitatory signal of fixed size $P$ to 


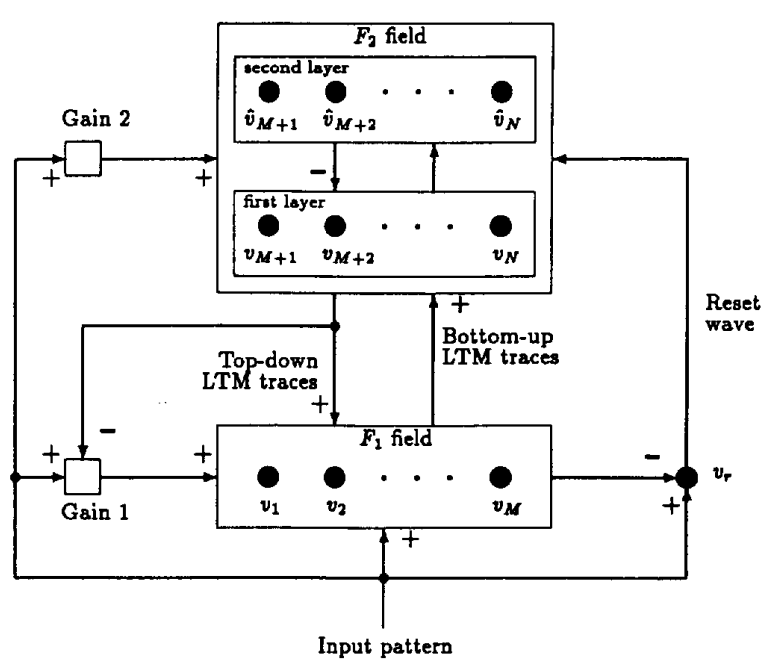

Fig. 2. The architecture of the augmented ART1 neural network model.

the orienting subsystem $A$, and each of the $|X|$ supraliminally active nodes in the $F_{1}$ field generates an inhibitory signal of fixed size $Q$ that also impinges on the orienting subsystem. Furthermore, the orienting subsystem in the ART1 model generates a nonspecific reset wave whenever

$$
P|I|>Q|X|
$$

or equivalently, whenever

$$
\frac{|X|}{|I|}<\rho=\frac{P}{Q}
$$

where $\rho$, the vigilance parameter, is chosen in the interval $(0,1]$.

The generation of the reset wave by the orienting subsystem can be accomplished within the framework of a dynamical system model through the introduction of a reset node, $v_{r}$, whose activity satisfies the following differential equation:

$$
\epsilon_{r} \frac{d}{d t} x_{r}=-A_{r} x_{r}+\mathrm{U}\left[P \sum_{i=1}^{M} I_{i}-Q \sum_{i=1}^{M} f_{1}\left(x_{i}\right)\right]
$$

where $\mathrm{U}$ is the unit step function

$$
\mathrm{U}(x)= \begin{cases}1, & \text { if } x>0 \\ 0 & \text { otherwise }\end{cases}
$$

Note that the activity of the reset node becomes positive whenever $\frac{|X|}{|\bar{I}|}<\rho$, and decays exponentially to zero whenever $\frac{|X|}{|I|} \geq \rho$. The output activity of the reset node, $f_{r}\left(x_{r}\right)$, which corresponds to the nonspecific reset wave, satisfies

$$
f_{r}\left(x_{r}\right)= \begin{cases}1, & \text { if } x_{r}>\delta_{r} \\ 0, & \text { otherwise }\end{cases}
$$

The introduction of a reset node whose activity satisfies (18), and whose output activity is determined by (20), provides a mechanism for the generation of the reset wave by the orienting subsystem, as required by the ART1 model, whenever there is a sufficient mismatch between the input pattern $I$ and the activity pattern $X$ across the $F_{1}$ field.
Resolution of Issue ii: An important property of the ART1 model is that the reset wave selectively and enduringly inhibits active $F_{2}$ field nodes until the input pattern is removed. This can be accomplished within the framework of a dynamical system realization of ART1 by augmenting the $F_{2}$ field with a set of inhibitory nodes (second layer of the $F_{2}$ field in Fig. 2), whose sole purpose is to implement the selective and enduring inhibition of the reset mechanism. In this case, every node $v_{j}$ in the $F_{2}$ field is assigned an inhibitory node $\hat{v}_{j}$ whose activity, $\hat{x}_{j}$, satisfies the following differential equation

$$
\epsilon_{2} \frac{d}{d t} \hat{x}_{j}=-[1-g(I)] \hat{x}_{j}+g(I) f_{r}\left(x_{r}\right) f_{2}\left(x_{j}\right)
$$

where

$$
g(I)= \begin{cases}1, & \text { if } \sum_{i=1}^{M} I_{i} \neq 0 \\ 0, & \text { otherwise. }\end{cases}
$$

As can be seen from the above equations, the activity of an $F_{2}$ field inhibitory node can only become positive when the following actions are satisfied simultaneously: a nonzero input pattern is being presented to the network, a reset wave is being emitted by the reset node, and the corresponding node in the $F_{2}$ field is supraliminally active. Once the activity of an $F_{2}$ field inhibitory node has become positive, its activity decays exponentially to zero only when the input pattern is removed. In conjunction with a modification to the differential equation characterizing the activity of the first layer of $F_{2}$ field nodes, this mechanism will allow the implementation of the selective and enduring inhibition required after a reset event, and as long as the input pattern is present. Specifically, the total inhibitory input to node $v_{j}$ in the $F_{2}$ field, $(10)$, is modified as

$$
J_{j}^{-}=\sum_{k \neq j} f_{2}\left(x_{k}\right)+\hat{f}_{2}\left(\hat{x}_{j}\right)
$$

where $\hat{f}_{2}\left(\hat{x}_{j}\right)$ is the output of the $F_{2}$ field inhibitory node $\hat{v}_{j}$. This modification causes the total inhibitory input to $v_{j}$ to remain positive as long as $\hat{f}_{2}\left(\hat{x}_{j}\right)$ is positive. The output of an $F_{2}$ field inhibitory node obeys the equation:

$$
\hat{f}_{2}\left(\hat{x}_{j}\right)= \begin{cases}1, & \text { if } \hat{x}_{j}>\hat{\delta}_{2} \\ 0, & \text { otherwise. }\end{cases}
$$

These modifications and additions to the original ART1-NN equations allow the $F_{2}$ field of the AART1-NN to behave as a gated dipole field within the dynamical systems framework.

Resolution of Issue iii: A modification to the equation describing the total excitatory input to an $F_{2}$ field node must also be made to allow the ART1 implementation to operate as a true dynamical system. The aforementioned modification will allow the activity of the $F_{1}$ and $F_{2}$ field nodes, as well as the activity of the reset node to be reset to zero whenever an input pattern is removed from the network. This can be accomplished in the following manner. When an input pattern is removed from the network, it should be followed by the presentation of the zero pattern. This will rapidly drive the activity of nodes in the $F_{1}$ and $F_{2}$ field to zero if we modify (7) as

$$
J_{j}^{+}=f_{2}\left(x_{j}\right) g(I)+D_{2} \sum_{i} f_{1}\left(x_{i}\right) z_{i j}
$$


TABLE I

Time Instances Pertaining to the Operation of the ART1-NN. The SUPERSCRIPT ASSOCIATED WiTH a SPECIFIC TIME INSTANCE RePRESENTS THE Number of Nodes in the $F_{2}$ Field that have been Sequentially activated During the Presentation of the Current Input Pattern

\begin{tabular}{|c||l|}
\hline Time Instance & \multicolumn{1}{|c|}{ Interpretation } \\
\hline$s_{0}$ & $\begin{array}{l}\text { All nodes are subliminally active, and a nonzero pattern } \\
\text { I is presented at the } F_{1} \text { field. }\end{array}$ \\
\hline$s_{1}^{\gamma-1}$ & The output pattern at the $F_{1}$ field is equal to $I$. \\
\hline$s_{2}^{\gamma}$ & $\begin{array}{l}\text { A single node, } v_{M+r} \text { in the } \widetilde{F}_{2} \text { field becomes supralimi- } \\
\text { nally active. }\end{array}$ \\
\hline$s_{3}^{\gamma}$ & The orienting subsystem generates a reset wave. \\
\hline$s_{1}^{\gamma}$ & Node $v_{M+r}$ in the $F_{2}$ field becomes subliminally active. \\
\hline$s_{5}$ & The input pattern $I$ is withdrawn from the $\mathscr{F}_{1}$ field. \\
\hline \multicolumn{2}{|l}{} \\
\hline
\end{tabular}

Conceptually, this presents no problems as it represents an absence of stimuli at the network inputs. That is, instead of a constant bombardment of stimuli, the learning system is allowed a brief "rest period" between each stimulus presentation. During this period, the activity of the network nodes are allowed to return to their resting values.

\section{ANALYSIS OF THE ART1 NEURAL NETWORK}

In the previous equations defining the AART1-NN model, the quantities $x_{i}, x_{j}, \hat{x}_{j}, J_{i}^{+}, J_{i}^{-}, J_{j}^{+}$, and $J_{j}^{-}$are functions of time; while the quantities $A_{1}, B_{1}, C_{1}, \epsilon_{1}, \delta_{1}, A_{r}, \epsilon_{r}, \delta_{r}$, $A_{2}, B_{2}, D_{2}, \epsilon_{2}, \delta_{2}, \hat{\delta}_{2}, K, L$, and $\epsilon_{z}$ are constants which we will refer to as the "parameters" of the AART1-NN. In this section we show that, under certain AART1-NN parameter constraints, the operation of the AART1-NN is identical to that of the ART1-NN. It is important to realize that the AART1NN parameter constraints developed below are sufficient, but not necessary conditions for the successful operation of the AART1-NN.

\section{A. Preliminaries}

We begin by defining a number of important time instances that can be identified during the operation of the ART1NN. These time instances, along with their corresponding definitions, are given in Table I. The use of these time instances allows us to concisely describe the behavior of the ART1NN during the presentation of a nonzero input pattern. Note that the superscript of a specific time instance identifies the number of nodes in the $F_{2}$ field of the ART1-NN that have been sequentially activated during the presentation of the input pattern. It should also be noted that the time instances in Table I correspond to the points on the time axis at which the state of at least one node in the ART1-NN changes from a subliminally active mode to a supraliminally active mode, or vice versa. The only exceptions are the time instances $\left\{s_{3}^{\gamma}\right\}$, which correspond to the points on the time axis at which the orienting subsystem generates a reset wave. These time instances are also important because a reset wave in the ART1-NN forces a supraliminally active node in the $F_{2}$ field to become subliminally active.

Assume that a nonzero input pattern $I$ is presented to the $F_{1}$ field of the ART1-NN, and consider the time instance $s_{1}^{0}$ at which the output activity across the $F_{1}$ field is equal to $I$. At this time instance, $O_{M+1}, O_{M+2}, \cdots, O_{N-1}$, and $O_{N}$, are defined to be the bottom-up inputs that affect the $F_{2}$ field nodes $v_{M+1}, v_{M+2}, \cdots, v_{N-1}$, and $v_{N}$, respectively. Without loss of generality we assume that $O_{M+1}>O_{M+2}>\cdots>O_{N-1}>$
$O_{N}$. We also assume that each one of these bottom-up inputs is large enough to activate an $F_{2}$ field node, if it is the only input affecting this node. We now distinguish two cases:

Case 1: During the presentation of the input pattern $I$ at the $F_{1}$ field of the ART1-NN, $\Gamma-1$ reset events occur, where $1 \leq \Gamma \leq N-M$. That is, the $\Gamma$-th activated node in the $F_{2}$ field codes $I$.

Case 2: During the presentation of the input pattern $I$ at the $F_{1}$ field of the ART1-NN, $\Gamma=N-M$ reset events occur. In this case, no node in the $F_{2}$ field is able to code $I$.

Let us now consider a nonzero input pattern $I$ belonging to the class of patterns described as Case 1 or Case 2 above. If $I$ is presented to the $F_{1}$ field of the ART1-NN, then the behavior of the network can be described by the following statement:

Statement 1: During the presentation of the nonzero input pattern $I$ to the $F_{1}$ field of the ART1-NN, the following time instances can be identified in order of occurrence:

Case 1: $s_{0}, s_{1}^{0}, s_{2}^{1}, s_{3}^{1}, s_{4}^{1}, s_{1}^{1}, s_{2}^{2}, s_{3}^{2}, s_{4}^{2}, \ldots, s_{1}^{\Gamma-3}, s_{2}^{\Gamma-2}$, $s_{3}^{\Gamma-2}, s_{4}^{\Gamma-2}, s_{1}^{\Gamma-2}, s_{2}^{\Gamma-1}, s_{3}^{\Gamma-1}, s_{4}^{\Gamma-1}, s_{1}^{\Gamma-1}, s_{2}^{\Gamma}, s_{5}$.

Case 2: $s_{0}, s_{1}^{0}, s_{2}^{1}, s_{3}^{1}, s_{4}^{1}, s_{1}^{1}, s_{2}^{2}, s_{3}^{2}, s_{4}^{2}, \ldots, s_{1}^{\Gamma-3}, s_{2}^{\Gamma-2}$, $s_{3}^{\Gamma-2}, s_{4}^{\Gamma-2}, s_{1}^{\Gamma-2}, s_{2}^{\Gamma-1}, s_{3}^{\Gamma-1}, s_{4}^{\Gamma-1}, s_{1}^{\Gamma-1}, s_{2}^{\Gamma}, s_{3}^{\Gamma}, s_{4}^{\Gamma}, s_{5}$.

A competition cycle in the ART1-NN is defined as the time period during which the nodes in the $F_{2}$ field, that have not yet been reset, compete in order to choose the node that most accurately represents the input pattern. In the implementation of the ART1-NN under consideration, the node which receives the largest bottom-up input, and has not been previously reset during the current pattern presentation, is chosen as the "winner." The first competition cycle starts at time $s_{1}^{0}$ and ends at time $s_{2}^{1}$, while the $\gamma$-th competition cycle $(\gamma \geq 2)$ starts at time $s_{4}^{\gamma-1}$ and ends at time $s_{2}^{\gamma}$. Note that fast learning corresponds to the case where the input pattern is presented long enough for the network to choose the node in the $F_{2}$ field that codes the input pattern, and furthermore, this pattern is held at the network inputs long enough for the bottomup and top-down LTM traces of this node to reach their limiting values. In contrast, slow learning corresponds to the case where the input pattern is presented long enough for the network to choose the node in the $F_{2}$ field that codes the input pattern, but not necessarily long enough for the bottom-up and top-down LTM traces of this node to reach their limiting values.

In terms of the behavior of the ART1-NN, we can define an equivalent set of time instances corresponding to the operation of AART1-NN. Specifically, the important time instances that can be identified during the operation of the AART1-NN are given in Table II. These time instances are equivalent to those given in Table I, except that they take into account the new elements incorporated into the AART1-NN. For example, instead of the time instances $\left\{s_{3}^{\gamma}\right\}$ of Table I which represent the times at which reset waves are generated in the ART1NN, Table II contains corresponding time instances $\left\{t_{3}^{\gamma}\right\}$ which represent the times at which the reset node $v_{r}$ becomes supraliminally active in the AART1-NN. Furthermore, since the $F_{2}$ field of the AART1-NN consists of two layers of nodes, any references in the terminology of the ART1-NN to nodes in the $F_{2}$ field are replaced with references to nodes in the first 
TABLE II

Time Instances Pertaining to the Operation of the AART1-NN. THE SupersCRIPT Associated With a SPECIFIC TIME InSTANCE REPrESENTS THE NUMBER OF NODES IN THE FIRST LAYER OF THE $F_{2}$ FiELD THAT haVE BEEN SEQuENTIALly ACTIVATED During the PRESEntation of THE CuRREnt InPUT PATtern

\begin{tabular}{|c|l|}
\hline Time Instance & \multicolumn{1}{c|}{ Interpretation } \\
\hline$t_{0}$ & $\begin{array}{l}\text { All nodes are subliminally active, and a nonzero input } \\
\text { pattern } I \text { is presented at the } F_{1} \text { field. }\end{array}$ \\
\hline$t_{1}^{\gamma-1}$ & The output pattern at the $F_{1}$ field is equal to $I$. \\
\hline$t_{2}^{\gamma}$ & $\begin{array}{l}\text { A single node, } v_{M+\gamma} \text {, in the first layer of the } F_{2} \text { field } \\
\text { becomes supraliminally active. }\end{array}$ \\
\hline$t_{3}^{\gamma}$ & The reset node $v_{r}$ becomes supraliminally active. \\
\hline$t_{4}^{\gamma}$ & $\begin{array}{l}\text { Node } v_{M+\gamma} \text { in the first layer of the } F_{2} \text { field becomes sub- } \\
\text { liminally active. }\end{array}$ \\
\hline$t_{5}$ & The input pattern $I$ is withdrawn from the $F_{1}$ field. \\
\hline
\end{tabular}

layer of the $F_{2}$ field. Thus, the superscript of a time instance defined in Table II corresponds to the number of nodes in the first layer of the $F_{2}$ field of the AART1-NN that have been sequentially activated during the presentation of input pattern I.

Because the time instances defined in Table II are equivalent to those defined in Table I, it follows that the behavior of the AART1-NN will be identical to that of the ART1-NN if it can be shown that the AART1-NN operates in a manner similar to Statement 1. Specifically, consider a nonzero input pattern $I$ belonging to the class of patterns described as Case 1 or Case 2 above. If $I$ is presented to the $F_{1}$ field of an AART1-NN, then the behavior of this network will be equivalent to that of an ART1-NN if the following statement holds:

Statement 2: During the presentation of the nonzero input pattern $I$ to the $F_{1}$ field of the AART1-NN, the following time instances can be identified in order of occurrence:

Case 1: $t_{0}, t_{1}^{0}, t_{2}^{1}, t_{3}^{1}, t_{4}^{1}, t_{1}^{1}, t_{2}^{2}, t_{3}^{2}, t_{4}^{2}, \cdots, t_{1}^{\Gamma-3}, t_{2}^{\Gamma-2}, t_{3}^{\Gamma-2}$, $t_{4}^{\Gamma-2}, t_{1}^{\Gamma-2}, t_{2}^{\Gamma-1}, t_{3}^{\Gamma-1}, t_{4}^{\Gamma-1}, t_{1}^{\Gamma-1}, t_{2}^{\Gamma}, t_{5}$.

Case 2: $t_{0}, t_{1}^{0}, t_{2}^{1}, t_{3}^{1}, t_{4}^{1}, t_{1}^{1}, t_{2}^{2}, t_{3}^{2}, t_{4}^{2}, \cdots, t_{1}^{\Gamma-3}, t_{2}^{\Gamma-2}, t_{3}^{\Gamma-2}$, $t_{4}^{\Gamma-2}, t_{1}^{\Gamma-2}, t_{2}^{\Gamma-1}, t_{3}^{\Gamma-1}, t_{4}^{\Gamma-1}, t_{1}^{\Gamma-1}, t_{2}^{\Gamma}, t_{3}^{\Gamma}, t_{4}^{\Gamma}, t_{5}$.

In this case, the first competition cycle starts at time $t_{1}^{0}$ and ends at time $t_{2}^{1}$, while the $\gamma$-th competition cycle $(\gamma \geq 2)$ starts at time $t_{4}^{\gamma-1}$ and ends at time $t_{2}^{\gamma}$. The fast and slow learning operations of the AART1-NN are defined in the same manner as they were for the ART1-NN.

In the next section we prove that under certain AART1NN parameter constraints, the AART1-NN behaves according to Statement 2. Consequently, we prove that under these parameter constraints, the behavior of the AART1-NN is identical to that of the ART1-NN. In the proof of Statement 2 we will assume, without loss of generality, that a nonzero input pattern $I$ belonging to the class of patterns described as Case 1 above is presented to the AART1-NN. We will then prove that during the presentation of this pattern, the time instances included in Statement 2 under Case 1 can be identified. The proof of Statement 2 is accomplished by demonstrating that with the appropriate parameter values, each time instance occurs in the order given. That is, we will show that if we start with time instance $t_{0}$, the next identifiable time instance is $t_{1}^{0}$, and if we start with time instance $t_{1}^{0}$ the next identifiable time instance is $t_{2}^{1}$, etc., until time instance $t_{5}$ is reached.
It should be emphasized that Statement 2 describes the behavior of the AART1-NN only when a nonzero input pattern is presented at its $F_{1}$ field. The operation of the AART1-NN during the presentation of the zero pattern is easily determined from the AART1-NN equations. It can be shown that the presentation of the zero pattern to the $F_{1}$ field of the AART1NN drives the activities of all the nodes in the network to zero. Since the zero pattern is always presented between the presentation of any two nonzero patterns, it follows that the AART1-NN equations satisfy a key ART1-NN design constraint, namely:

- ART1 design constraint \#1: The activities of all the network nodes in the ART1-NN should be reset to zero prior to the presentation of any nonzero input pattern at its $F_{1}$ field.

During the proof of Statement 2, the AART1-NN parameter values will be chosen so as to satisfy a number of additional ART1 design constraints given below:

- ART1 design constraint \#2: The input pattern $I$ must be able to instate itself across the $F_{1}$ field without triggering a reset event, at least until an $F_{2}$ field node becomes active and sends top-down signals to the $F_{1}$ field.

- ART1 design constraint \#3: The order of the $O_{j}$ 's (i.e., the bottom-up inputs to the $F_{2}$ field) determine the order of search in the $F_{2}$ field, no matter how many times the $F_{2}$ field is reset.

- ART1 design constraint \#4: During the presentation of the input pattern $I$ at the $F_{1}$ field, a node in the $F_{2}$ field that wins a competition cycle can only be reset if there is sufficient mismatch between bottom-up and top-down inputs.

ART1 design constraint \#1 is explicitly stated in [1], while ART1 design constraint \#2 is explicitly stated in [17] as one of the fundamental ART design constraints. Finally, ART1 design constraints \#3 and \#4 are stated in [1] as Theorem 3 and Corollary 1 , respectively.

\section{B. Proof of Statement 2}

In this section we prove Statement 2. To facilitate this proof we present in Appendix A a set of key AART1-NN equations. Before we proceed with the proof, let us first refer to a number of constraints that are important for the successful operation of the ART1-NN, and as a result for the successful operation of the AART1-NN. These are constraints CON1-CON7 included in Table III.

CON1 requires the STM values in the AART1-NN to change at a much faster rate than the LTM values. Furthermore, the requirement $\epsilon_{r}<<\epsilon_{z}$ implies that the reset node in the AART1-NN responds very quickly (compared to the LTM changes in the network) to mismatches between bottom-up and top-down inputs at the $F_{1}$ field of the AART1-NN. CON1 guarantees that no significant LTM learning occurs in the AART1-NN unless the node picked in the $F_{2}$ field of the AART1-NN is the node that codes the input pattern. An immediate implication of CON1 is that it allows us to assume that the LTM traces in the AART1-NN stay constant from the time that the input pattern is presented, until the time that 
TABLE III

CONSTRAINTS FOR SUCCESSFUL OPERATION OF THE AART1-NN

\begin{tabular}{|c|c|}
\hline CON1 & $\epsilon_{1}, \epsilon_{2}, \epsilon_{r} \ll<\epsilon_{z}$ \\
\hline CON2 & $A_{1}>0, C_{1}>0, \quad A_{2}>0, \quad C_{2}>0$ \\
\hline CON3 & $B_{1}>0, D_{1}>0, B_{2}>0, D_{2}>0$ \\
\hline CON4 & $0<\rho \leq 1$ \\
\hline CON5 & $L>1,0<z_{i j}(0)<L(L-1+M)^{-1}$ \\
\hline CON6 & {$\left[B_{1}-1+\delta_{1}\left(1+A_{1}+C_{1}\right)\right]\left[D_{1}\left(1-\delta_{1} A_{1}\right)\right]^{-1}<z_{j i}(0) \leq 1$} \\
\hline CON7a & $\max \left\{1, D_{1}\right\}-\delta_{1}\left(1+A_{1}+C_{1}\right)<B_{1}$ \\
\hline CON7b & $B_{1}<1+D_{1}-\delta_{1}\left(1+A_{1}+D_{1} A_{1}+C_{1}\right)$ \\
\hline CON8 & 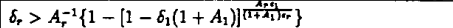 \\
\hline CON9 & $B_{2}>O_{\max }$ \\
\hline CON10 & $\overline{B_{2}}>1+O_{\max }$ \\
\hline CoN11a & $C_{2} \gg 1+A_{2} O_{\max }$ \\
\hline CON116 & $B_{2}=p_{1} O_{\max }^{2}, C_{2}=p_{1} O_{\max }$ \\
\hline CON11c & $B_{2} C_{2}^{-1} \gg \delta_{2}$ \\
\hline CONi1d & $p_{3}^{\delta_{3}}<<1$ \\
\hline CON12 & $p_{4} \approx \delta_{1}$ \\
\hline CON13 & $\epsilon_{1} \leq 0.1 \epsilon_{2}, \quad A_{2} O_{\max }<A_{1}$ \\
\hline CON14 & $O_{M+n+1}<p_{7} O_{M+n}$ \\
\hline CON15 & $-p_{5} O_{\max } p_{\mathrm{g}}+\left(1-p_{\mathrm{g}}\right) O_{\max }\left(1+A_{2} O_{\max }\right)^{-1}<\delta_{2}$ \\
\hline CON16 & $\left(1+A_{1}\right)^{-1}>\delta_{1}$ \\
\hline CON17 & \\
\hline
\end{tabular}

the node in the first layer of its $F_{2}$ field that codes the input pattern is chosen. CON2 guarantees that the activity $x_{i}$ will be constrained in the interval $\left[-B_{1} C_{1}^{-1}, A_{1}^{-1}\right]$, and that the activity $x_{j}$ will be constrained in the interval $\left[-B_{2} C_{2}^{-1}, A_{2}^{-1}\right]$. $\mathrm{CON} 3$, and the fact that the $z_{i j}$ 's and $z_{j i}$ 's are nonnegative (see constraints CON5, CON6, CON7, and (13) and (14)), ensures that $J_{i}^{+}$and $J_{j}^{+}$are indeed excitatory signals, and that $J_{i}^{-}$and $J_{j}^{-}$are indeed inhibitory signals. CON4 is required for the successful operation of the reset mechanism. CON5 is important for the satisfaction of the direct access inequality-for more details see [1]. CON6 and CON7 are necessary for the validity of the $\frac{2}{3}$ rule (see equation (A.2) in Appendix A)

We now proceed with the proof of Statement 2 . Consider a nonzero input pattern $I$ which is presented to the $F_{1}$ field of the AART1-NN, and assume without loss of generality that this pattern belongs to the class of patterns previously described as Case 1. We will prove that under certain AART1-NN parameter constraints, the time instances included in Statement 2 under Case 1 can be identified. This validates Statement 2 for Case 1, and obviously for Case 2 as well.

The Time Interval After $t_{0}$ : We begin by assuming that a nonzero input pattern $I$ is presented to the $F_{1}$ field of the AART1-NN at time instance $t_{0}$. The activities of all the nodes in the $F_{1}$ field are equal to zero at $t_{0}$. It is easy to see that we can identify a time, after $t_{0}$, at which the output pattern at the $F_{1}$ field is equal to $I$. We have already denoted the earliest such time instance as $t_{1}^{0}$. During the time interval $\left(t_{0}, t_{1}^{0}\right]$ the activity of every node at the $F_{2}$ field is equal to zero. Furthermore, during the time interval $\left(t_{0}, t_{1}^{0}\right)$, there is mismatch at the $F_{1}$ field. This is due to the fact that the pattern $I$ has not yet been instated across the $F_{1}$ field. The parameters of the AART1NN must be chosen so as to satisfy ART1 design constraint \#2. To satisfy this constraint it suffices to choose the AART1$\mathrm{NN}$ parameters so that the reset node is subliminally active at time instance $t_{1}^{0}$. In Appendix B we show that by choosing the AART1-NN parameters according to CON8 in Table III, we ensure that the reset node $v_{r}$ is subliminally active at time instance $t_{1}^{0}$.

The Time Interval After $t_{1}^{0}$ : At time instance $t_{1}^{0}$ a node $v_{j}$ in the $F_{2}$ field receives bottom-up input $O_{j}$ from the $F_{1}$ field, and satisfies equation (A.3) after time instance $t_{1}^{0}$. In (A.3), $t_{a}$ corresponds to $t_{1}^{0}$ and $T_{j}$ corresponds to $O_{j}$. Since every node in the first layer of the $F_{2}$ field has an activity of zero at $t_{1}^{0}$, and $T_{j}=O_{j}$, we can identify a time, after $t_{1}^{0}$, at which the only node in the first layer of the $F_{2}$ field that is supraliminally active is $v_{M+1}$-we have already denoted this time instance as $t_{2}^{1}$. As a result, ART1 design constraint \#3 is satisfied in the first competition cycle (i.e., in the interval $\left.\left(t_{1}^{0}, t_{2}^{1}\right]\right)$. In the time interval $\left(t_{1}^{0}, t_{2}^{1}\right]$ the reset node satisfies equation (A.7) with $t_{a}=t_{1}^{0}$. Note that by choosing the AART1-NN parameters according to CON8 we guarantee that $x_{r}\left(t_{1}^{0}\right)<\delta_{r}$. Hence, at $t_{2}^{1}$ the reset node $v_{r}$ is still subliminally active. Thus, ART1 design constraint \#4 is also valid in the first competition cycle. Note that at time $t_{2}^{1}$ there is no mismatch between bottom-up and top-down inputs at the $F_{1}$ field.

The Time Interval After $t_{2}^{1}$ : After time instance $t_{2}^{1}$ every node $v_{j}(j \neq M+1)$ satisfies equation (A.4) with $t_{a}=t_{2}^{1}$. Note that $x_{j}\left(t_{2}^{1}\right)<\delta_{2}$ for $j \neq M+1$. Let us choose the parameter $B_{2}$ according to CON9 of Table III, where $O_{\max }$ is an upper bound on the $O_{j}$ 's for any input pattern presented to the AART1-NN. Choosing $B_{2}$ according to constraint CON9 guarantees that no other node in the first layer of the $F_{2}$ field becomes supraliminally active as long as $v_{M+1}$ is supraliminally active. In the time interval after $t_{2}^{1}$, certain nodes in the $F_{1}$ field receive bottom-up, as well as top-down input. An arbitrary node $v_{i}$ in the $F_{1}$ field that receives bottomup and weak top-down input will become subliminally active some time after $t_{2}^{1}$. Let us assume, without loss of generality, that enough nodes in the $F_{1}$ field receive bottom-up and weak top-down input so as to cause a positive input to the reset node. Once these nodes become subliminally active, the activity of the reset node will satisfy (A.6), and the reset node will generate a reset wave at some time after $t_{2}^{1}$. We have previously denoted this time instance as $t_{3}^{1}$.

The Time Interval After $t_{3}^{1}$ : The reset wave initiated at time instance $t_{3}^{1}$ will cause an excitatory input to be supplied to the inhibitory node $\hat{v}_{M+1}$. This results in the activation of $\hat{v}_{M+1}$, which in turn produces an inhibitory input to node $v_{M+1}$. From this time instance, node $v_{M+1}$ in the $F_{2}$ field will satisfy (A.5). Let us choose parameter $B_{2}$ according to CON10 of Table III. The satisfaction of CON10 guarantees that the supraliminally active node $v_{M+1}$ will become subliminally active at some time after $t_{3}^{1}$. We have previously denoted this time instance as $t_{4}^{1}$.

The Time Interval After $t_{4}^{1}$ : At time instance $t_{4}^{1}$, the previously supraliminally active node $v_{M+1}$ is subliminally active, and it will stay subliminally active as long as the input pattern is present. The deactivation of node $v_{M+1}$ signals the beginning of the second competition cycle. Once $v_{M+1}$ becomes subliminally active, the activities of all subliminally active nodes in the $F_{1}$ field that receive bottom-up input will start to increase. The output activity at the $F_{1}$ field is now changing from $X \subset I$ to $X=I$. The satisfaction of ART1 design constraint \#3 during the second competition cycle is not a trivial matter - as it was when we were examining the first competition cycle. This is because the competition cycle starts at time instance $t_{4}^{1}$, and from $t_{4}^{1}$ until the time that the output activity across the $F_{1}$ field becomes equal to $I$, the bottom-up 
inputs $T_{M+2}$ and $T_{j}(j \neq M+1, M+2)$ can be different from the bottom-up inputs $O_{M+2}$ and $O_{j}(j \neq M+1, M+2)$ that should determine the order of search in the AART1-NN. To make this point clearer, we present in Appendix $\mathrm{C}$ an extreme case where $T_{M+2}=0$ and $T_{M+3}=O_{M+3}$. To ensure that the AART1-NN does not perform the search in an erroneous order (e.g., a search of node $v_{M+3}$ prior to the search of node $v_{M+2}$ for the example in Appendix $C$ ) we require that the output activity across the $F_{1}$ field be restored to $I$ long before the second competition cycle ends. Thus, the AART1-NN will have sufficient time to overcome the detrimental effects of a situation where $T_{M+2}<T_{j}(j \neq M+1, M+2)$ at the beginning of the second competition cycle. We denote this requirement as Requirement A. Below we develop a set of AART1-NN parameter constraints for satisfying Requirement A.

Let us assume that Requirement $A$ is true. We will now prove that this assumption is valid under certain AART1NN parameter constraints. Consider a node $v_{i}$ that receives a bottom-up input from the input pattern $I$, and is subliminally active at time instance $t_{4}^{1}$. After $t_{4}^{1}$ the activity of this node satisfies (A.1) with $t_{a}=t_{4}^{1}$ and $x_{i}\left(t_{4}^{1}\right)<\delta_{1}$. If we assume that among the nodes in the $F_{1}$ field that are subliminally active at $t_{4}^{1}$, node $v_{1}$ becomes supraliminally active last, we can write

$$
t_{1}^{1}-t_{4}^{1}=\ln \left[\frac{x_{1}\left(t_{4}^{1}\right)\left(1+A_{1}\right)-1}{\delta_{1}\left(1+A_{1}\right)-1}\right]\left(1+A_{1}\right)^{-1} \epsilon_{1} .
$$

In (26), $t_{1}^{1}$ denotes the time that the output activity across the $F_{1}$ field is equal to $I$. Consider now a node $v_{j}(j \neq M+1)$ at time instance $t_{4}^{1}$. Let us denote by $x_{j}^{u}(t)$ an upper bound for the activity of node $v_{j}$, for $t$ in the time interval $\left(t_{4}^{1}, t_{1}^{1}\right]$. Note that $x_{j}^{u}(t)$ satisfies (A.3) with $t_{a}=t_{4}^{1}, x_{j}^{u}\left(t_{4}^{1}\right)=x_{j}\left(t_{4}^{1}\right)<\delta_{2}$, and $T_{j}=O_{j}$. The reason that $x_{j}^{u}(t)$ is an upper bound on $x_{j}(t)$ for $t \in\left(t_{4}^{1}, t_{1}^{1}\right]$ is because in this time interval, the bottom-up input $T_{j}$ to node $v_{j}$ increases towards its maximum value of $O_{j}$, and $O_{j}$ is attained at time instance $t_{1}^{1}$. It is obvious that our assumption regarding the validity of Requirement $\mathrm{A}$ will be true if the quantity $\delta_{2}-x_{j}^{u}\left(t_{1}^{1}\right)(j \neq M+1)$ is positive, and in fact as large as possible. The quantity $x_{j}^{u}\left(t_{1}^{1}\right)$ is given by (see (26) and (A.3)):

$$
\begin{aligned}
& x_{j}^{u}\left(t_{1}^{1}\right)=x_{j}\left(t_{4}^{1}\right) \Psi_{1}^{\frac{\left(1+A_{2} O_{j}\right) \epsilon_{1}}{\left(1+A_{1}\right) \epsilon_{2}}}+\frac{O_{j}}{1+A_{2} O_{j}} \\
& \times\left\{1-\Psi_{1} \frac{\left(1+A_{2} O_{j}\right) \epsilon_{1}}{\left(1+A_{1}\right) \epsilon_{2}}\right\}
\end{aligned}
$$

with

$$
\Psi_{1}=\frac{1-\delta_{1}\left(1+A_{1}\right)}{1-x_{1}\left(t_{4}^{1}\right)\left(1+A_{1}\right)} .
$$

One way of making the quantity $\delta_{2}-x_{j}^{u}\left(t_{1}^{1}\right)$ as large as possible is to force $x_{j}^{u}\left(t_{1}^{1}\right)$ to be as small as possible. Thus, we choose the AART1-NN parameters so that the value of $x_{j}\left(t_{4}^{1}\right)$ is approximately equal to its minimum value of $-B_{2} C_{2}^{-1}$. In Appendix D we show that this can be achieved by choosing the AART1-NN parameter values according to CON11 in Table III. Since CON11 guarantees that $x_{j}\left(t_{4}^{1}\right)$ is negative, we can now choose the AART1-NN parameters as in CON12 and CON13. CON12 guarantees that the value of
$\Psi_{1}$ is close to one (note that $x_{1}\left(t_{4}^{1}\right)$ is lower bounded by $p_{4}$ and upper bounded by $\delta_{1}$ ), while CON13 guarantees that the exponent of $\Psi_{1}$ is small. Constraint CON11, which implies $x_{j}\left(t_{4}^{1}\right) \approx-B_{2} C_{2}^{-1}=-O_{\max }$ (this is proven in Appendix D), in conjunction with $\mathrm{CON} 12$ and CON13 prove the validity of Requirement $\mathrm{A}$.

At time instance $t_{1}^{1}$, the output activity across the $F_{1}$ field is equal to $I$. The AART1-NN must satisfy ART1 design constraint \#3 in the second competition cycle that started at time instance $t_{4}^{1}$. The implication of ART1 design constraint $\# 3$ in this competition cycle is that node $v_{M+2}$ will be the first node activated after time instance $t_{1}^{1}$. In Appendix E we show that under constraint CON14 of Table III, ART1 design constraint \#3 is satisfied in the second competition cycle. The time of activation of node $v_{M+2}$ was previously denoted by time instance $t_{2}^{2}$. The index $n$ in CON14 corresponds to the index of the competition cycle under consideration. For example, since we are now interested in the second competition cycle, $n=2$. It is worth noting from Table III that $p_{5} \approx 1$ (due to CON12 and CON13), and as a result $p_{7} \approx 1$. This implies that CON14 does not actually impose hard constraints on the $O_{j}$ values. It is also worth noting that we do not have complete control over the $O_{j}$ values-they depend, among other things, on the set of input patterns. The reason that ART1 design constraint \#3 is satisfied under the mild conditions on the $O_{j}$ 's imposed by CON14 is because we previously guaranteed the satisfaction of Requirement $\mathrm{A}$.

We must also satisfy ART1 design constraint \#4 during the second competition cycle. The satisfaction of this design constraint in the second competition cycle requires that the reset node be subliminally active at time $t_{2}^{2}$. This is due to the fact that at time $t_{2}^{2}$, there is no mismatch between bottomup and top-down inputs at the $F_{1}$ field. We know that the reset node in the AART1-NN is supraliminally active at time instance $t_{4}^{1}$. We also know that it might be subliminally active at time instance $t_{1}^{1}$. If the reset node is subliminally active at time $t_{1}^{1}$, then we immediately satisfy ART1 design constraint \#4 in the second competition cycle, because we know that the reset node will be subliminally active at time $t_{2}^{2}$ as well. If on the contrary, the reset node is supraliminally active at time instance $t_{1}^{1}$, we need to guarantee that it will become subliminally active by time $t_{2}^{2}$. In Appendix $\mathrm{F}$ we show that this is indeed true under constraint CON15 of Table III. Hence, we can state that ART1 design constraint \#4 is valid in the second competition cycle provided that $\mathrm{CON} 15$ is satisfied.

The Time Intervals After $t_{2}^{\gamma}, 2 \leq \gamma \leq \Gamma-1$ : For every $\gamma$ such that $2 \leq \gamma \leq \Gamma-1$ we can show that $t_{3}^{\gamma}$ is the next identifiable time instance after time instance $t_{2}^{\gamma}$. The approach is similar to the one followed after time interval $t_{2}^{1}$.

The Time Intervals After $t_{3}^{\gamma}, 2 \leq \gamma \leq \Gamma-1$ : For every $\gamma$ such that $2 \leq \gamma \leq \Gamma-1$ we can show that $t_{4}^{\gamma}$ is the next identifiable time instance after time instance $t_{3}^{\gamma}$. The approach is similar to the one followed after time interval $t_{3}^{1}$.

The Time Intervals After $t_{4}^{\gamma}, 2 \leq \gamma \leq \Gamma-1$ : For every $\gamma$ such that $2 \leq \gamma \leq \Gamma-1$ we can show that $t_{1}^{\gamma}$ is the next identifiable time instance after time instance $t_{4}^{\gamma}$, and we can also show that $t_{2}^{\gamma+1}$ is the next identifiable time instance after time instance $t_{1}^{\gamma}$. The approach is similar to the one followed 
after time interval $t_{4}^{1}$. The only difference now is that we are dealing with different competition cycles (i.e., competition cycles $3,4, \cdots, \Gamma$ ).

Thus, after time $t_{2}^{2}$, time instances occur in the order prescribed by Statement 2 under Case 1, and no additional AART1-NN parameter constraints are necessary beyond those already developed (i.e., CON1-CON15). It is worth pointing out that CON14 depends on the index $n$ of the competition cycle under consideration. For Case 1 , which is under investigation, the range of $n$ is over the set $\{2,3, \cdots, \Gamma\}$. For Case 2 though, the range of $n$ is over the set $\{2,3, \cdots, N-M-1\}$. Since our objective is to guarantee the validity of Statement 2 under both cases, we take the range of $n$ to be the set $\{2,3, \cdots, N-M-1\}$.

The Time Interval After $t_{2}^{\Gamma}$ : At time instance $t_{2}^{\Gamma}$ the $\Gamma$-th node in the first layer of the $F_{2}$ field has been activated, and this node codes the input pattern $I$ (Case 1). LTM learning takes place after time instance $t_{2}^{\Gamma}$. It is obvious that $t_{5}$ is the next identifiable instance after time instance $t_{2}^{\Gamma}$. This time instance is designated as the time at which the input pattern $I$ is withdrawn from the $F_{1}$ field of the AART1-NN. If the interval $\left[t_{2}^{\Gamma}, t_{5}\right]$ is long enough, then we are dealing with the fast learning case; otherwise we are dealing with the slow learning case.

It should be mentioned that additional AART1-NN parameter constraints were implied throughout the proof of Statement 2. Specifically, constraints CON16 and CON17 of Table III. These constraints impose a lower bound on the forcing terms affecting (A.1) and (A.6). If the forcing terms in these equations do not exceed the lower bound, then the activities of the $F_{1}$ field and reset nodes will never exceed their quenching thresholds.

Concluding, we can state that we have derived constraints CON1-CON17 of Table III under which we proved the validity of Statement 2 . The validity of Statement 2 proves that the AART1-NN is capable of behaving in a manner identical to that of the ART1-NN.

\section{Parameter Choices}

Now that we have developed the AART1-NN parameter constraints listed in Table III, it is instructive to demonstrate how parameter values can be chosen for an example network. But first we mention some estimates of the quantities $O_{\max }$ and $O_{\min }$ that appear in CON1-CON17 of Table III. $O_{\max }$ is an upper bound on the $O_{j}$ 's for any pattern $I$ that is presented at the $F_{1}$ field of the AART1-NN. A loose estimate of $O_{\max }$ is $D_{2} M$. A better estimate of $O_{\max }$ is desirable; otherwise, CON11b of Table III will require unreasonably large values for the parameters $B_{2}$ and $C_{2}$. In the fast learning case it can be shown that a better estimate of $O_{\max }$ is $\frac{D_{2} L M}{L-1+M}$. In the slow learning case, and under the assumption that $L=1+\sigma$ with $\sigma<<1$, it can be shown that a better estimate of $O_{\max }$ is $D_{2}$. $O_{\min }$ is a lower bound on the $O_{j}$ 's for any input pattern $I$ that is presented at the $F_{1}$ field of the AART1-NN. Unless we have a better estimate for $O_{\min }$, based on some prior knowledge of the set of input patterns considered, it is always safe to take $O_{\min }=0$ in CON1-CON17.
The sample network considered here contains four nodes in the $F_{1}$ field (nodes $v_{1}$ through $v_{4}$ ), a reset node (node $v_{r}$ ), and eight nodes in the $F_{2}$ field (nodes $v_{5}$ through $v_{8}$ in the first layer, and inhibitory nodes $\hat{v}_{5}$ through $\hat{v}_{8}$ in the second layer). Consequently for the sample network $M=4$ and $N-M=4$. We initially choose $L=1.01$. This yields the estimate $O_{\max }=$ $D_{2}$. Subsequently, we choose $A_{1}=1, D_{1}=1, \epsilon_{1}=0.001$, $\delta_{1}=0.01, \epsilon_{r}=0.001, D_{2}=1, \epsilon_{2}=0.01, \delta_{2}=0.01$, and $\epsilon_{z}=1$. These parameters can be thought of as being the "free parameters" in the network, despite the fact that they have to satisfy constraints CON1-CON17. We refer to them as "free parameters" due to the fact that they are picked first.

Now we choose the remaining AART1-NN parameter values so as to satisfy CON1-CON17. We first choose $B_{1}$ and $C_{1}$ values to satisfy $\operatorname{CON} 2, \mathrm{CON} 3, \mathrm{CON} 7$ and $\mathrm{CON} 12$; the reader can verify that $B_{1}=0.5$ and $C_{1}=100$ satisfy these constraints. We then choose $A_{2}=0.3$, having in mind CON2, CON13 and CON14. Furthermore, we choose $B_{2}$ and $C_{2}$ in a way that satisfies constraints CON2, CON3, CON9, CON10, and CON11. In our example, we chose $B_{2}=10000$ and $C_{2}=10000$; it is easy for the reader to verify that CON2, CON3, CON9, CON10, and CON11 are satisfied. Finally, we pick $\delta_{r}=0.02$ and $A_{r}=2$ so as to satisfy CON8, CON15, and CON17. Based on the aforementioned AART1-NN parameter values, we chose the initial bottom-up traces, the $z_{i j}(0)$ 's, in the interval $(0,0.251)$ and the initial top-down traces, the $z_{j i}(0)$ 's, in the interval $(0.526,1]$ (see CON5 and CON6). As a rule of thumb, in order to satisfy AART1-NN parameter constraints CON1-CON17, we choose the parameter values to make $p_{3}$ and $e_{6}$ as small as possible; $p_{8}, e_{2}$, and $e_{3}$ as large as possible; $p_{5}, p_{6}$, and $p_{7}$ as close to one as possible; $p_{4}$ as close to $\delta_{1}$ as possible; and finally, $p_{2}$ as much larger than $\delta_{1}$ as possible.

The AART1-NN parameter values chosen for this example are listed in Table IV. Some of the parameter values did not have any effect on the successful operation of the network, and as a result were chosen arbitrarily (e.g., $\hat{\delta}_{2}=0.0001, K=1$ ). Furthermore the vigilance parameter $\rho$ was selected to be equal to 1 . Note that the AART1-NN should operate successfully for all the values of the vigilance parameter designated in CON4. Once more, the AART1-NN parameter values listed in Table IV satisfy all the constraints of Table III. Note though that the test for the validity of CON14 is computationally intensive for the fast learning case and almost impossible for the slow learning case. But it is worth observing that for the parameters chosen (i.e., $A_{2}=0.3$, and $O_{\max }=1$ ) CON14 is satisfied if $O_{M+n+1}<0.99979 O_{M+n}$ for $n=2, \cdots, N-M-1$. Thus, in this case, the AART1-NN will satisfy CON14 for most $O_{j}$ values of interest. Observe also that the ART1-NN, as defined in [1], operates successfully only when the $O_{j}$ 's are distinct.

\section{COMPUTER Simulation}

In this section we demonstrate the behavior of the AART1 model for both the fast and slow learning cases. As mentioned in Section $\mathrm{V}$, the sample network considered here contains four nodes in the $F_{1}$ field (nodes $v_{1}$ through $v_{4}$ ), a reset node (node $v_{r}$ ), and eight nodes in the $F_{2}$ field (nodes $v_{5}$ 
TABLE IV

AART1-NN PARAmeter VALUES FOR THE EXAMPLE NeTwork

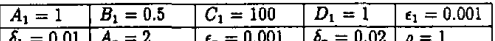
\begin{tabular}{|l|l|l|l|l|l|}
\hline$\delta_{1}=0.01$ & $A_{r}=2$ & $\epsilon_{r}=0.001$ & $\delta_{r}=0.02$ & $\rho=1$ \\
\hline$A_{2}=0.3$ & $B_{1}=10000$ & $C_{r}=1000$ & $D_{2}=1$ & $G_{3}=0.01$ \\
\hline
\end{tabular} \begin{tabular}{|l|l|l|l|}
$B_{2}=10000$ & $C_{2}=1000$ & $D_{2}=1$ & $\epsilon_{2}=0.01$ \\
\hline & $\delta_{2}=0.001$ & $\epsilon_{2}=1$ \\
\hline
\end{tabular} $\delta_{2}=0.01 \quad \delta_{2}=0.001 \quad K=1 \quad t \quad t=1.01 \quad G_{4}=1$

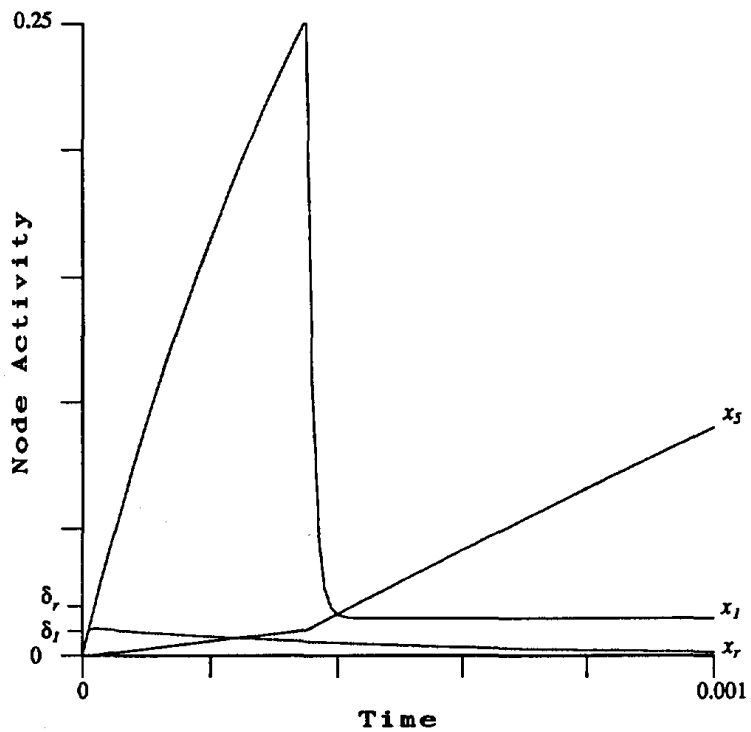

Fig. 3. Node activities during the presentation of pattern $I^{1}$. The sudden drop in activity $x_{1}$ is a manifestation of the $2 / 3$ rule in ART1.

through $v_{8}$ in the first layer, and inhibitory nodes $\hat{v}_{5}$ through $\hat{v}_{8}$ in the second layer). The node differential equations were numerically approximated using the fourth order Runge-Kutta method with a step size of $10^{-6}$. Three patterns were presented to the network: $I^{1}=1000, I^{2}=0000$, and $I^{3}=1100$. Note that $I^{2}$ is the zero pattern used between presentation of other "interesting" patterns. That is, the presentation of pattern $I^{2}$ can be interpreted as the absence of an input pattern. The parameters chosen for the simulation of the sample network in both the fast and slow learning cases are shown in Table IV. The LTM traces for these simulations were selected so that $z_{j i}(0)=1$, and $0<z_{i j}(0)<\frac{L}{L-1+M}$ for all $i, j$. In addition, the bottom-up LTM traces were chosen so that when pattern $I^{1}$ is initially presented, $v_{5}$ receives the largest bottomup input. Furthermore, when pattern $I^{3}$ is initially presented, $v_{5}$ receives the largest bottom-up input, and $v_{6}$ receives the next largest bottom-up input.

The behavior of the AART1-NN during the presentation of patterns $I^{1}, I^{2}$, and $I^{3}$ is described with reference to Figs. 3-7, which depict node activities versus time. Although time is a continuous parameter, it also has a meaning in terms of the number of steps elapsed during the approximation of the network differential equations. For the network simulations described in this section, time $t$ corresponds to $10^{6} \cdot t$ steps.

The fast learning case is examined first. Pattern $I^{1}=1000$ is presented to the network at time $t=0$. The behavior of the network immediately following the presentation of $I^{1}$ is depicted in Fig. 3. Among the $F_{1}$ field nodes, only $v_{1}$ is of interest because it is the only node receiving bottom-up input. After the input pattern is presented, the activity of $v_{1}$ increases from zero to a positive value above the threshold $\delta_{1}$. Once $v_{1}$ becomes supraliminally active, nodes in the first layer of the $F_{2}$ field begin to receive bottom-up input. Because $v_{5}$ receives the largest bottom-up input, it becomes supraliminally active before any other node in the first layer of the $F_{2}$ field. At this point, $v_{1}$ is receiving both bottom-up input, and strong top-down input from $v_{5}$. This causes the activity of $v_{1}$ to decrease and subsequently reach a limiting value that is above the threshold $\delta_{1}$. Thus, node $v_{1}$ remains supraliminally active. Furthermore, the activity of $v_{5}$ continues to increase over the time interval depicted in Fig. 3. Recall that once $v_{5}$ becomes supraliminally active, it will inhibit the other nodes in the first layer of the $F_{2}$ field, forcing them to remain subliminally active as long as it remains supraliminally active. The activity of the reset node $v_{r}$ in Fig. 3 should also be noted. Immediately after the presentation of $I^{1}$, the activity $x_{r}$ increases due to the mismatch between the output activity across the $F_{1}$ field, which equals zero, and the input pattern $I^{1}$. Notice that the output activity across the $F_{1}$ field becomes equal to $I^{1}$ before the activity of the reset node exceeds its threshold $\delta_{r}$. From this point on, the activity of the reset node decays towards its limiting value of zero. That is, even after the activation of $v_{5}$, the activity $x_{r}$ continues to decrease, due to the fact that there is no mismatch between bottom-up and top-down inputs across the $F_{1}$ field. Pattern $I^{1}$ is presented until time $t=3.0$. This allows the bottom-up and top-down LTM traces to approximately reach their limiting values.

At time $t=3.0$ pattern $I^{2}=0000$ is presented to the network. The behavior of the network after the appearance of pattern $I^{2}$ is shown in Fig. 4. Initially $v_{1}$ is at an activity level above the threshold $\delta_{1}$, but its activity drops to a level below $\delta_{1}$ almost instantaneously. This results from $v_{1}$ receiving only top-down input (prior to time $t=3.0$ it was receiving bottomup and strong top-down input). After the deactivation of $v_{1}$, the activity levels of nodes $v_{1}$ and $v_{2}$ stay at a constant level until $v_{5}$ is deactivated. The activity $x_{1}$ is larger than the activity $x_{2}$ because node $v_{1}$ receives stronger top-down input than $v_{2}$. In the meantime, the activity of $v_{5}$ drops from a positive value to zero. Once $v_{5}$ becomes subliminally active, the activities of $v_{1}$ and $v_{2}$ decrease to zero because they are no longer receiving top-down input. The activity of $v_{6}$ starts increasing from a negative value towards zero immediately after $v_{5}$ becomes subliminally active. The behavior of $v_{6}$ is not fully depicted in Fig. 4 because its activity is significantly negative $(\approx-1.0)$ when $v_{5}$ becomes subliminally active. Pattern $I^{2}$ is held at the network input until time $t=3.2$.

At time $t=3.2$ pattern $I^{3}=1100$ is presented. The behavior of the network after the presentation of $I^{3}$ is depicted in Figs. 5 and 6. In Fig. 5, after the presentation of $I^{3}, v_{5}$ becomes supraliminally active before any other node in the first layer of the $F_{2}$ field because it receives the largest bottomup input from the $F_{1}$ field. Once $v_{5}$ becomes supraliminally active, the activities of nodes $v_{1}$ and $v_{2}$ begin to decrease. The activity of $v_{1}$ remains above the threshold $\delta_{1}$, while the activity of $v_{2}$ decreases to a level below $\delta_{1}$. This is a consequence of $v_{1}$ receiving strong top-down input, while $v_{2}$ 


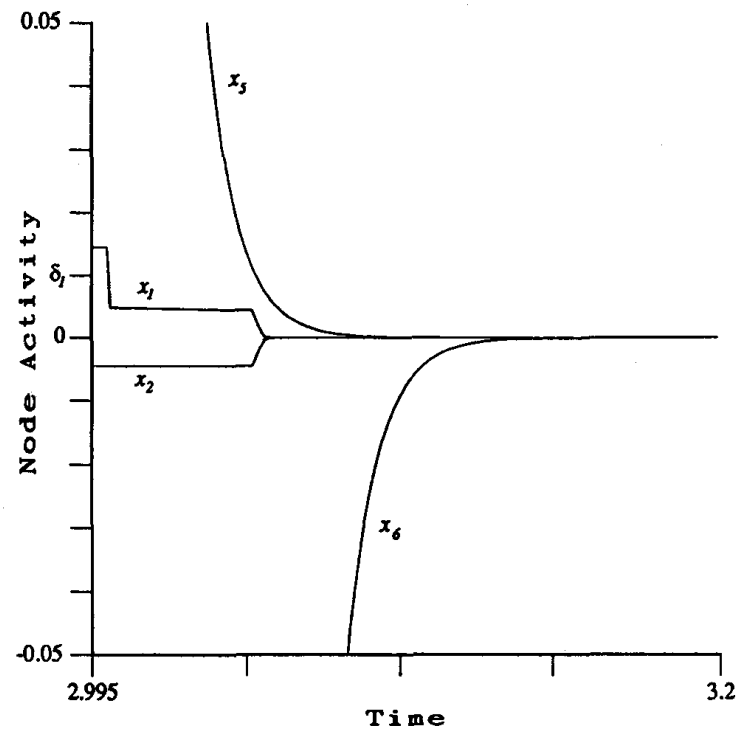

Fig. 4. Node activities during the presentation of pattern $I^{2}$.

receives weak top-down input-both nodes receive bottom-up input. When $v_{2}$ becomes subliminally active, the activity of the reset node starts increasing due to the mismatch between the bottom-up and top-down inputs that is now occurring at the $F_{1}$ field. When $v_{r}$ becomes supraliminally active (i.e., its activity exceeds $\delta_{r}$ ) it generates a reset wave that deactivates $v_{5}$ almost instantaneously. After $v_{5}$ becomes subliminally active, $v_{1}$ and $v_{2}$ receive only bottom-up input, and their activities increase towards the limiting value of 0.5 (see Fig. 6). Now that $v_{5}$ is deactivated, $v_{6}$ will become supraliminally active next since it is the node in the first layer of the $F_{2}$ field that receives the next largest bottom-up input from the $F_{1}$ field. That is, $v_{6}$ will be activated before any other eligible node $\left(v_{7}\right.$ or $\left.v_{8}\right)$ in the first layer of the $F_{2}$ field. The activation of $v_{6}$ is shown in Fig. 6. When $v_{6}$ becomes supraliminally active, the activities of nodes $v_{1}$ and $v_{2}$ begin to decrease from the value 0.5 ; but they remain above the quenching threshold $\delta_{1}$. This is a consequence of both $v_{1}$ and $v_{2}$ receiving bottom-up input and strong top-down input. Notice also that the activity of the reset node starts decreasing some time after the deactivation of $v_{5}$ (see Fig. 5), and that it continues to do so after the activation of $v_{6}$ (see Fig. 6) because there is no mismatch between bottomup and top-down inputs at the $F_{1}$ field. Hence, when pattern $I^{3}$ is held at the network inputs long enough, the bottom-up and top-down LTM traces reach their limiting values.

We now consider the slow learning case. First, pattern $I^{1}$ is presented at time $t=0$, and the network exhibits the behavior depicted in Fig. 3. However, in this case, soon after $v_{5}$ wins the competition in the first layer of the $F_{2}$ field, pattern $I^{1}$ is removed from the network inputs. Thus, the bottom-up and top-down LTM traces are not allowed to converge to their limiting values. Pattern $I^{1}$ is presented until time $t=0.1$, and then pattern $I^{2}=0000$ is presented. By time $t=0.3$, all node activities have converged to their resting values of zero. The behavior of the network during the presentation of

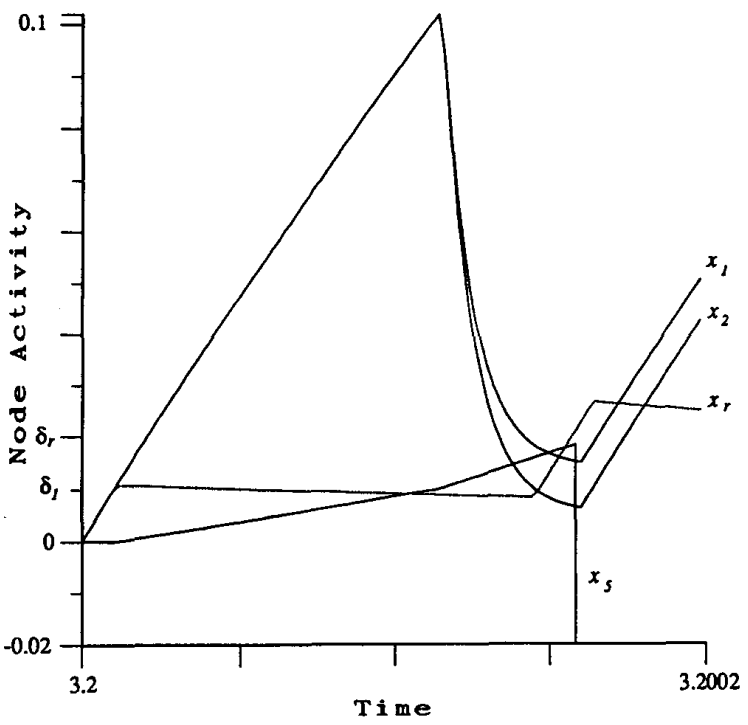

Fig. 5. Node activities leading to a reset during the presentation of pattern $I^{3}$. The sudden drop in activities $x_{1}$ and $x_{2}$ is again a manifestation of the $2 / 3$ rule in ART1. Note that the activity of node $v_{1}$ remains supraliminally active, while node $v_{2}$ becomes subliminally active. Because of the choice of the $\rho$ parameter, this causes the activity of the reset node $v_{r}$ to become supraliminally active, and leads to a reset of node $v_{5}$.

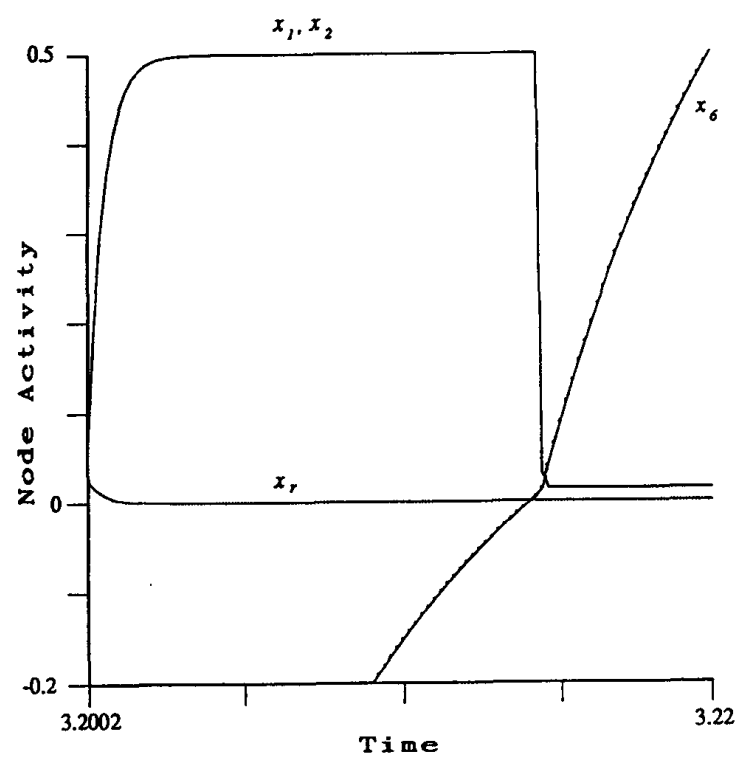

Fig. 6. Node activities after the reset during the presentation of pattern $I^{3}$.

pattern $I^{2}$ is similar to that shown in Fig. 4, with the time instances 3.0 and 3.2 now corresponding to time instances 0.1 and 0.3 . The major difference between the fast and slow learning cases demonstrated in these simulations occurs when pattern $I^{3}$ is presented to the network at time $t=0.3$. The behavior of the network after the presentation of pattern $I^{3}$ is depicted in Fig. 7. It is instructive to compare Figs. 5 and 6 , the network behavior in the fast learning case after pattern $I^{3}$ is presented, with Fig. 7. As in the fast learning case, $v_{5}$ 


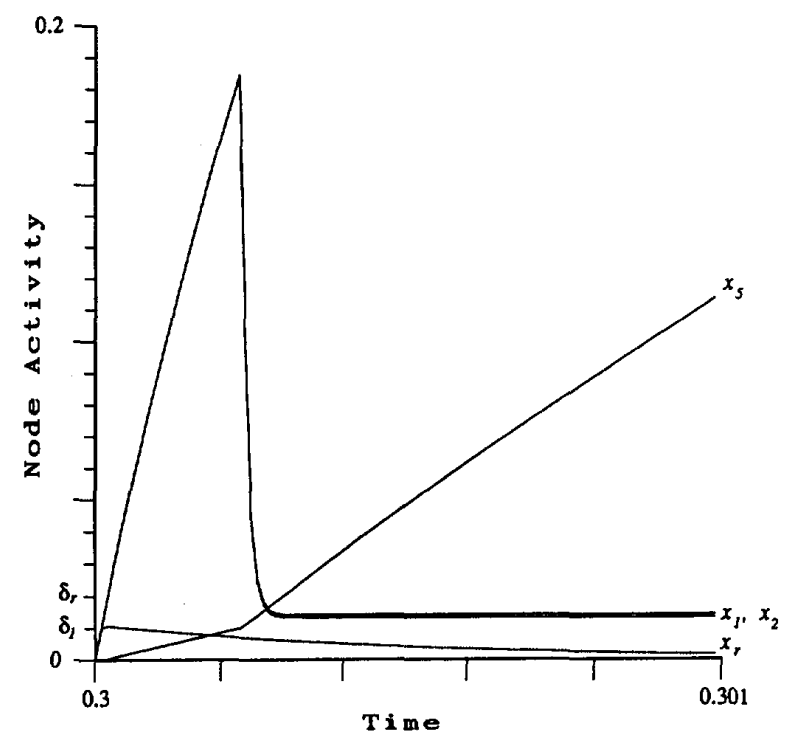

Fig. 7. Node activities after the presentation of pattern $I^{3}$, when pattern $I^{1}$ has not been coded by $v_{5}$ on a previous pattern presentation.

receives the largest bottom-up input. Hence, $v_{5}$ is activated prior to any other node in the first layer of the $F_{2}$ field. This activation forces the activities of nodes $v_{1}$ and $v_{2}$ to decrease to limiting values that remain above the threshold value $\delta_{1}$. In the slow learning case, the fact that $x_{2}$ remains above $\delta_{1}$ while pattern $I^{3}$ is presented is a consequence of not allowing the top-down traces leading to $v_{5}$ to approach their equilibrium values during the presentation of pattern $I^{1}$. As a result, when $v_{5}$ becomes supraliminally active, nodes $v_{2}$ and $v_{1}$ receive bottom-up input and strong top-down input. Thus, since both $v_{1}$ and $v_{2}$ stay supraliminally active, $v_{5}$ is not reset. That is, the reset node remains subliminally active throughout the time that pattern $I^{3}$ is presented. Therefore, if pattern $I^{3}$ is held at the network inputs long enough, the LTM traces of node $v_{5}$ will approach their limiting values.

\section{CONCLUSIONS}

The contribution of our work is twofold. First, we extended the ART1-NN model by both introducing new, and modifying already existing ART1 differential equations. This dynamical system model was denoted the AART1-NN. The distinguishing feature of the AART1-NN is that it incorporates all of the ART1 mechanisms into a set of coupled nonlinear differential equations. Second, we rigorously analyzed the AART1-NN equations and showed that they exhibit an ART1-NN behavior, as it is documented in [1, sections 3-6]. Although it is implied in [1] that the ART1 model can be implemented in this fashion, a thorough justification is not provided. This work demonstrates how the ART1 model can be cast into the form of a nonlinear dynamical system, and supplies a method for proving that this dynamical system will exhibit the behavior of the ART1 model. Furthermore, the capability of implementing the ART1 model in this fashion is of practical importance because it allows the tools used in the analysis of nonlinear dynamical systems to be applied (see the discussion in Section I). Finally, the method applied to analyze the STM and LTM dynamics of the AART1-NN can be extended to other neural network models where the network dynamics are described via a set of nonlinear differential equations.

\section{APPENDIX A}

In this appendix we present a number of AART1-NN equations that are instrumental to the proof of Statement 2. The activity of a node $v_{i}$ that receives bottom-up input, and no top-down input, satisfies the following equation for $t \in\left(t_{a}, t_{b}\right]$ :

$$
\begin{aligned}
x_{i}(t)= & x_{i}\left(t_{a}\right) \exp \left[-\left(1+A_{1}\right) \epsilon_{1}^{-1}\left(t-t_{a}\right)\right] \\
& +\frac{1}{1+A_{1}}\left[1-\exp \left[-\left(1+A_{1}\right) \epsilon_{1}^{-1}\left(t-t_{a}\right)\right]\right] .
\end{aligned}
$$

The above equation is obtained by solving equation (2) in the main text with $J_{i}^{+}=1$ and $J_{i}^{-}=0$.

The activity of a node $v_{i}$ that receives bottom-up input, as well as top-down input from a node $v_{j}$ satisfies the following equation for $t \in\left(t_{a}, t_{b}\right)$ :

$$
\begin{aligned}
x_{i}(t)= & x_{i}\left(t_{a}\right) \exp \left[-\left(1+A_{1}+D_{1} A_{1} z_{j i}+C_{1}\right) \epsilon_{1}^{-1}\left(t-t_{a}\right)\right] \\
& +\frac{1+D_{1} z_{j i}-B_{1}}{1+A_{1}+D_{1} A_{1} z_{j i}+C_{1}} \\
& \times\left[1-\exp \left[-\left(1+A_{1}+D_{1} A_{1} z_{j i}+C_{1}\right) \epsilon_{1}^{-1}\left(t-t_{a}\right)\right]\right] .
\end{aligned}
$$

The above equation is derived by solving (2) with $J_{i}^{+}=$ $1+D_{1} z_{j i}$ and $J_{i}^{-}=1$. In the above equation, the case where $\frac{1+D_{1} z_{j i}-B_{1}}{1+A_{1}+D_{1} A_{1} z_{i j}+C_{1}}>\delta_{1}$ can be distinguished from the case where $\frac{1+D_{1} z_{j i}-B_{1}}{1+A_{1}+D_{1} A_{1} z_{j i}+C_{1}} \leq \delta_{1}$. In the former case, we say that node $v_{i}$ receives bottom-up input and strong top-down input from node $v_{j}$. In the latter case, we say that node $v_{i}$ receives bottom-up input and weak top-down input from node $v_{j}$.

The activity of a node $v_{j}$ that receives a bottom-up input $T_{j}$ from the $F_{1}$ field over a time interval in which no node in the first layer of the $F_{2}$ field is supraliminally active, satisfies the following equation for $t \in\left(t_{a}, t_{b}\right]$ :

$$
\begin{aligned}
x_{j}(t)= & x_{j}\left(t_{a}\right) \exp \left[-\left(1+A_{2} T_{j}\right) \epsilon_{2}^{-1}\left(t-t_{a}\right)\right] \\
& +\frac{T_{j}}{1+A_{2} T_{j}}\left[1-\exp \left[-\left(1+A_{2} T_{j}\right) \epsilon_{2}^{-1}\left(t-t_{a}\right)\right]\right] .
\end{aligned}
$$

The above equation is obtained by solving (6) with $J_{j}^{+}=T_{j}$ and $J_{j}^{-}=0$.

The activity of a node $v_{j}$ that receives a bottom-up input $T_{j}$ from the $F_{1}$ field over a time interval in which another node in the first layer of the $F_{2}$ field is supraliminally active, satisfies the following equation for $t \in\left(t_{a}, t_{b}\right]$ :

$$
\begin{aligned}
x_{j}(t)= & x_{j}\left(t_{a}\right) \exp \left[-\left(1+A_{2} T_{j}+C_{2}\right) \epsilon_{2}^{-1}\left(t-t_{a}\right)\right] \\
& +\frac{T_{j}-B_{2}}{1+A_{2} T_{j}+C_{2}} \\
& \times\left[1-\exp \left[-\left(1+A_{2} T_{j}+C_{2}\right) \epsilon_{2}^{-1}\left(t-t_{a}\right)\right]\right] .
\end{aligned}
$$


The above equation is derived by solving (6) with $J_{j}^{+}=T_{j}$ and $J_{j}^{-}=1$.

The activity of the only supraliminally active node $v_{j}$ that receives a bottom-up input $T_{j}$ from the $F_{1}$ field satisfies, after the initiation of a reset wave by the reset node $v_{r}$, the following equation:

$\frac{d}{d t} x_{j}=-\left(1+A_{2}+A_{2} T_{j}+C_{2}\right) \epsilon_{2}^{-1} x_{j}+\left(1+T_{j}-B_{2}\right) \epsilon_{2}^{-1}$.

The above equation is derived from (6) by substituting $J_{j}^{+}$ with $1+T_{j}$ and $J_{j}^{-}$with 1 .

The activity of the reset node $v_{r}$, if there is a mismatch between the input pattern $I$ and the output activity across the $F_{1}$ field, satisfies the following equation for $t \in\left(t_{a}, t_{b}\right]$ :

$$
\begin{aligned}
x_{r}(t)= & x_{r}\left(t_{a}\right) \exp \left[-A_{r} \epsilon_{r}^{-1}\left(t-t_{a}\right)\right] \\
& +\frac{1}{A_{r}}\left[1-\exp \left[-A_{r} \epsilon_{r}^{-1}\left(t-t_{a}\right)\right]\right] .
\end{aligned}
$$

The above equation is obtained by solving (18) with

$$
\mathrm{U}\left[P \sum_{i=1}^{M} I_{i}-Q \sum_{i=1}^{M} f_{1}\left(x_{i}\right)\right]=1 .
$$

If there is no mismatch between $I$ and the output activity across the $F_{1}$ field over this interval, then the activity of the reset node satisfies

$$
x_{r}(t)=x_{r}\left(t_{a}\right) \exp \left[-A_{r} \epsilon_{r}^{-1}\left(t-t_{a}\right)\right] .
$$

The above equation is obtained by solving (18) with

$$
\mathrm{U}\left[P \sum_{i=1}^{M} I_{i}-Q \sum_{i=1}^{M} f_{1}\left(x_{i}\right)\right]=0 .
$$

\section{APPENDIX B}

In this appendix we show that by choosing the AART1NN parameter values according to CON8 we can guarantee that the reset node is subliminally active at time instance $t_{1}^{0}$. The activity of a node $v_{i}$ that receives bottom-up inputs in the interval $\left(t_{0}, t_{1}^{0}\right]$ satisfies $(A .1)$ with $t_{a}=t_{0}$ and $x_{i}\left(t_{a}\right)=$ $x_{i}\left(t_{0}\right)=0$. At time instance $t=t_{1}^{0}, x_{i}(t)=x_{i}\left(t_{1}^{0}\right)=\delta_{1}$. Thus,

$$
t_{1}^{0}-t_{0}=\frac{-\ln \left\{1-\delta_{1}\left(1+A_{1}\right)\right\} \epsilon_{1}}{1+A_{1}} .
$$

Since the activity of the reset node $v_{r}$ in the time interval $\left(t_{0}, t_{1}^{0}\right]$ satisfies (A.6) with $t_{a}=t_{0}$ and $x_{r}\left(t_{a}\right)=x_{r}\left(t_{0}\right)=0$, the activity of the reset node at time instance $t=t_{1}^{0}$ is given by

$$
x_{r}\left(t_{1}^{0}\right)=\frac{1-\left[1-\delta_{1}\left(1+A_{1}\right)\right]^{\frac{A_{r} \epsilon_{1}}{\left(1+A_{1}\right) \epsilon_{r}}}}{A_{r}} .
$$

Therefore, by choosing the AART1-NN parameter values as in CON8 we can guarantee that the reset node is subliminally active at time instance $t_{1}^{0}$.

\section{APPENDIX C}

In this appendix we provide an example where in the second competition cycle, corresponding to the presentation of an input pattern $I$ at the $F_{1}$ field of the AART1-NN, $T_{M+2}=0$ and $T_{M+3}=O_{M+3}$. The network under consideration consists of eight nodes in the $F_{1}$ field (i.e., nodes $v_{1}$ through $v_{8}$ ), and four nodes in the first and second layer of the $F_{2}$ field (i.e., nodes $v_{9}$ through $v_{12}$ in the first layer, and nodes $\hat{v}_{9}$ through $\hat{v}_{12}$ in the second layer). Consequently, in this sample network $M=8$ and $N-M=4$. The vigilance parameter $\rho$ is chosen to be 1 . Let us assume that the input patterns are presented long enough at the $F_{1}$ field of the AART1-NN so that fast LTM learning occurs. Let us also assume that prior to the presentation of the input pattern $I$, the network has already learned the input patterns $I_{1}=00001111, I_{2}=$ 01110000 , and $I_{3}=00001100$. In particular, the LTM bottomup and top-down traces of node $v_{9}$ are equal to $0000 a a a a$ and 00001111 , respectively, where $a=L\{L-1+4\}^{-1}$. Furthermore, the LTM bottom-up and top-down traces of node $v_{10}$ are equal to $0 b b b 0000$ and 01110000 , respectively, where $b=L\{L-1+3\}^{-1}$. Finally, the LTM bottom-up and topdown traces of node $v_{11}$ are equal to $0000 c c 00$ and 00001100 , where $c=L\{L-1+2\}^{-1}$. In short, node $v_{9}$ has learned the input pattern $I_{1}$, node $v_{10}$ has learned the input pattern $I_{2}$, and node $v_{11}$ has learned the input pattern $I_{3}$.

We now present pattern $I=01111111$ at the $F_{1}$ field of the AART1-NN. We assume that the network parameters are chosen so that $O_{9}>O_{10}>O_{11}>O_{12}$. As a result, node $v_{9}$ in the first layer of the $F_{2}$ field will be activated first, and it will be reset since $\rho=1$. Time instance $t_{4}^{1}$ has been designated as the time at which node $v_{9}$ is deactivated; at this time the second competition cycle starts. In the time interval $\left(t_{4}^{1}, t_{1}^{1}\right)$, it is easy to see that the output activity across the $F_{1}$ field is equal to 00001111 . Consequently, in the time interval $\left(t_{4}^{1}, t_{1}^{1}\right)$ the bottom-up input $T_{10}$ (i.e., $T_{M+2}$ ) is equal to zero, while the bottom-up input $T_{11}$ (i.e., $T_{M+3}$ ) is equal to $O_{M+3}$. This demonstrates our point.

\section{APPENDIX D}

\section{Appendix D.1}

In this appendix we prove that by choosing the AART1NN parameter values according to CON11 we can guarantee that $x_{j}\left(t_{4}^{1}\right)$ in (27) is approximately equal to its minimum value of $-B_{2} C_{2}^{-1}$. Let us examine the interval $\left(t_{2}^{1}, t_{4}^{1}\right]$. Within this interval there is at least one node in the $F_{1}$ field that receives bottom-up and weak top-down signals. We assume, without loss of generality, that node $v_{1}$ is the first node in the $F_{1}$ field that becomes subliminally active after time instance $t_{2}^{1}$. Let us denote by $t_{2}^{1}+\Delta t_{1}$ the time at which node $v_{1}$ becomes subliminally active. In the time interval $\left(t_{2}^{1}, t_{2}^{1}+\Delta t_{1}\right)$, the activity of the node $v_{j} ;(j \neq M+1)$ satisfies (A.4) with $t_{a}=t_{2}^{1}$ and $T_{j}=O_{j}$. If we show that at time $t_{2}^{1}+\Delta t_{1}$ the activity of a node $v_{j} ;(j \neq M+1)$ is, by appropriately choosing the AART1-NN parameters, upperbounded by $\varepsilon \theta+\left(-B_{2} C_{2}^{-1}\right)(1-\theta)$ for $\varepsilon$ and $\theta$ small positive constants, and $\varepsilon$ small compared to $B_{2} C_{2}^{-1}$, then we have 
shown that $x_{j}\left(t_{4}^{1}\right)$ is approximately equal to its minimum value of $-B_{2} C_{2}^{-1}$. This is true because the activity of a node $v_{j}(j \neq M+1)$ continues to decrease after time $t_{2}^{1}+\Delta t_{1}$ and until time $t_{4}^{1}$, and because the activities of all the nodes in the first layer of the $F_{2}$ field are lower bounded by $-B_{2} C_{2}^{-1}$.

Let us first determine $\Delta t_{1}$. In the time interval $\left(t_{2}^{1}, t_{2}^{1}+\Delta t_{1}\right)$ the activity of the node $v_{1}$ satisfies (A.2) with $t_{a}=t_{2}^{1}$, and $z_{j i}=z_{M+1,1}$. The quantity $\Delta t_{1}$ is found by setting $x_{1}\left(t_{2}^{1}+\Delta t_{1}\right)$ equal to $\delta_{1}$ in (A.2). This yields

$$
\Delta t_{1}=\frac{\ln \left(\Psi_{2}\right) \epsilon_{1}}{1+A_{1}+D_{1} A_{1} z_{M+1,1}+C_{1}}
$$

with

$\Psi_{2}=$

$\frac{-\left(1+D_{1} z_{M+1,1}-B_{1}\right)+x_{1}\left(t_{2}^{1}\right)\left(1+A_{1}+D_{1} A_{1} z_{M+1,1}+C_{1}\right)}{-\left(1+D_{1} z_{M+1,1}-B_{1}\right)+\delta_{1}\left(1+A_{1}+D_{1} A_{1} z_{M+1,1}+C_{1}\right)}$.

Furthermore, the activity of node $v_{j}(j \neq M+1)$ at time $t_{2}^{1}+\Delta t_{1}$ will be given by (A.4) if we substitute $x_{j}\left(t_{a}\right)$ with $x_{j}\left(t_{2}^{1}\right), t-t_{a}$ with $\Delta t_{1}$ from above, and $T_{j}$ with $O_{j}$. This yields

$$
\begin{aligned}
x_{j}\left(t_{2}^{1}+\Delta t_{1}\right)= & x_{j}\left(t_{2}^{1}\right) \Psi_{3} \frac{\left(1+A_{2} O_{j}+C_{2}\right) \epsilon_{1}}{\left(1+A_{1}+D_{1} A_{1}{ }^{z} M+1,1+C_{1}\right) \epsilon_{2}} \\
& +\frac{O_{j}-B_{2}}{1+A_{2} O_{j}+C_{2}} \\
& \times\left\{1-\Psi_{3}^{\frac{\left(1+A_{2} O_{j}+C_{2}\right) \epsilon_{1}}{\left(1+A_{1}+D_{1} A_{1}{ }_{1} M+1,1+C_{1}\right) \epsilon_{2}}}\right\}
\end{aligned}
$$

where $\Psi_{3}=\Psi_{2}^{-1}$. Since $\Psi_{3}$ is greater than zero and $x_{j}\left(t_{2}^{1}\right)<\delta_{2} ;(j \neq M+1)$, an upper bound for the right hand side of (D.1) is found if $x_{j}\left(t_{2}^{\frac{1}{2}}\right)$ is substituted with its upper bound $\delta_{2}$. Furthermore, let us choose the AART1-NN parameters in a way that allows us to approximate the term $\left(O_{j}-B_{2}\right)\left(1+A_{2} O_{j}+C_{2}\right)^{-1}$ with the term $-B_{2} C_{2}^{-1}$. It is not difficult to see that we can accomplish this goal by choosing the AART1-NN parameters according to CON11a and CON1lb in Table III. Consequently, we can now state that

$$
\begin{aligned}
x_{j}\left(t_{2}^{1}+\Delta t_{1}\right)< & \delta_{2} \Psi_{3}^{\frac{\left(1+A_{2} O_{j}+C_{2}\right) \epsilon_{1}}{\left(1+A_{1}+D_{1} A_{1} z_{M+1,1}+C_{1}\right) \epsilon_{2}}}+\left(-B_{2} C_{2}^{-1}\right) \\
& \times\left\{1-\Psi_{3}^{\frac{\left(1+A_{2} O_{j}+C_{2}\right) \epsilon_{1}}{\left(1+A_{1}+D_{1} A_{1} z_{M+1,1}+C_{1} \epsilon_{2}\right.}}\right\} .
\end{aligned}
$$

In Appendix D.2 we show that $\Psi_{3}$ decreases as $z_{M+1,1}$ increases. Furthermore, in Appendix D.3 we demonstrate that $x_{1}\left(t_{2}^{1}\right)$ is lower bounded by $p_{2}$, where $p_{2}$ is given in Table $\mathrm{V}$. These two facts allow us to state that $\Psi_{3}$ is upper bounded by $p_{3}$ which is also defined in Table V. Finally, it is easy to see that the exponent of $\Psi_{3}$ in (D.2) is lower bounded by the value for $e_{3}$ given in Table V. Combining all of the aforementioned facts we have from (D.2) that

$$
x_{j}\left(t_{2}^{1}+\Delta t_{1}\right)<\delta_{2} p_{3}^{e_{3}}+\left(-B_{2} C_{2}^{-1}\right)\left(1-p_{3}^{e_{3}}\right) .
$$

The upper bound on $x_{j}\left(t_{2}^{1}+\Delta t_{1}\right)$ in (D.3) is in the desired form, provided that CON11c and CON11d are satisfied. In
TABLE V

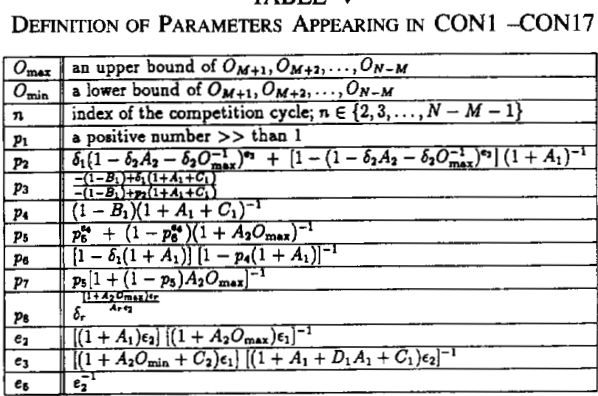

review, we have demonstrated in this appendix that under CON11 we can state that $x_{j}\left(t_{4}^{1}\right) \approx-B_{2} C_{2}^{-1}=-O_{\max } ;(j \neq$ $M+1)$.

\section{Appendix D.2:}

In this appendix we show that $\Psi_{3}$, defined in Appendix D.1, decreases as $z_{M+1,1}$ increases. The quantity $\Psi_{3}$ was defined in Appendix D.1 as

$\Psi_{3}=$

$\frac{-\left(1+D_{1} z_{M+1,1}-B_{1}\right)+\delta_{1}\left(1+A_{1}+D_{1} A_{1} z_{M+1,1}+C_{1}\right)}{-\left(1+D_{1} z_{M+1,1}-B_{1}\right)+x_{1}\left(t_{2}^{1}\right)\left(1+A_{1}+D_{1} A_{1} z_{M+1,1}+C_{1}\right)}$.

Let us evaluate the derivative of $\Psi_{3}$ with respect to $z_{M+1,1}$. The derivative of $\Psi_{3}$ with respect to $z_{M+1,1}$ is a ratio. The denominator of this ratio is a positive number. If the numerator of this ratio is negative then we have proven that $\Psi_{3}$ decreases as $z_{M+1,1}$ increases. Let us examine the numerator of $\frac{d \Psi_{3}}{d z_{M+1,1}}$ :

$$
\begin{aligned}
( & \left.-D_{1}+\delta_{1} D_{1} A_{1}\right)\left[-\left(1+D_{1} z_{M+1,1}-B_{1}\right)\right. \\
+ & \left.x_{1}\left(t_{2}^{1}\right)\left(1+A_{1}+D_{1} A_{1} z_{M+1,1}+C_{1}\right)\right] \\
+ & \left(D_{1}-x_{1}\left(t_{2}^{1}\right) D_{1} A_{1}\right)\left[-\left(1+D_{1} z_{M+1,1}-B_{1}\right)\right. \\
+ & \left.\delta_{1}\left(1+A_{1}+D_{1} A_{1} z_{M+1,1}+C_{1}\right)\right] \\
= & {\left[x_{1}\left(t_{2}^{1}\right)-\delta_{1}\right]\left\{-D_{1}\left(1+A_{1}+D_{1} A_{1} z_{M+1,1}+C_{1}\right)\right.} \\
& \left.+D_{1} A_{1}\left(1+D_{1} z_{M+1,1}-B_{1}\right)\right\} \\
< & {\left[x_{1}\left(t_{2}^{1}\right)-\delta_{1}\right]\left\{-D_{1}\left(1+A_{1}+D_{1} A_{1} z_{M+1,1}+C_{1}\right)\right.} \\
& \left.+\delta_{1} D_{1} A_{1}\left(1+A_{1}+D_{1} A_{1} z_{M+1,1}+C_{1}\right)\right\} \\
= & D_{1}\left[x_{1}\left(t_{2}^{1}\right)-\delta_{1}\right]\left(\delta_{1} A_{1}-1\right)\left(1+A_{1}+D_{1} A_{1} z_{M+1,1}+C_{1}\right) .
\end{aligned}
$$

In the last expression derived above, $D_{1}>0, x_{1}\left(t_{2}^{1}\right)-\delta_{1}>$ 0 (because node $v_{1}$ is supraliminally active at time $t_{2}^{1}$ ), $\delta_{1} A_{1}-1<0\left(A_{1}^{-1}\right.$ is the maximum activation value of a node in the $F_{1}$ field, and $\delta_{1}$ is the threshold of the node), and $1+A_{1}+D_{1} A_{1} z_{M+1,1}>0$. Consequently, the numerator of the derivative $\frac{d \Psi_{3}}{d z_{M+1,1}}$ is negative, which proves that $\Psi_{3}$ decreases as $z_{M+1,1}$ increases. The inequality utilized in the above derivations is justified because node $v_{1}$ is by assumption a node that receives bottom-up and weak top-down inputs, and as a result $1+D_{1} z_{M+1,1}-B_{1}<\left(1+A_{1}+D_{1} A_{1} z_{M+1,1}+\right.$ $\left.C_{1}\right) \delta_{1}$.

\section{Appendix D.3:}

In this appendix we prove that $x_{1}\left(t_{2}^{1}\right) \geq p_{2}$, where $p_{2}$ is defined in Table V. Consider the interval $\left(t_{1}^{0}, t_{2}^{1}\right]$. In this 
interval, the activity of every node $v_{i}$ that receives bottom-up input, and hence the activity of node $v_{1}$, satisfies (A.1) with $t_{a}=t_{1}^{0}$. Furthermore, in this interval, the activity of every node $v_{j}$ satisfies (A.3) with $t_{a}=t_{1}^{0}$ and $T_{j}=O_{j}$. Since node $v_{M+1}$ wins this competition cycle, we can write

$$
t_{2}^{1}-t_{1}^{0}=\frac{\ln \left(\Psi_{4}\right) \epsilon_{2}}{1+A_{2} O_{M+1}}
$$

with

$$
\Psi_{4}=\frac{O_{M+1}-x_{M+1}\left(t_{1}^{0}\right)\left(1+A_{2} O_{M+1}\right)}{O_{M+1}-\delta_{2}\left(1+A_{2} O_{M+1}\right)}
$$

Due to the above equations, the activity of node $v_{1}$ at time instance $t_{2}^{1}$ is given by (A.1) if we substitute $t_{a}$ with $t_{1}^{0}$, and $t-t_{a}$ with $t_{2}^{1}-t_{1}^{0}$ from above. This yields

$$
\begin{aligned}
x_{1}\left(t_{2}^{1}\right)= & x_{1}\left(t_{1}^{0}\right) \Psi_{5}^{\frac{\left(1+A_{1}\right) \epsilon_{2}}{\left(1+A_{2} O_{M+1}\right) \epsilon_{1}}}+\frac{1}{1+A_{1}} \\
& \times\left\{1-\Psi_{5}^{\frac{\left(1+A_{1}\right) \epsilon_{2}}{\left(1+A_{2} O_{M+1}\right) \epsilon_{1}}}\right\},
\end{aligned}
$$

where $\Psi_{5}=\Psi_{4}^{-1}$. We are now ready to derive a lower bound for $x_{1}\left(t_{2}^{1}\right)$ of equation (D.4). Because $x_{1}\left(t_{1}^{0}\right)>\delta_{1}$ and $\Psi_{5}>0$, a lower bound on the right hand side of (D.4) can be found if we substitute $x_{1}\left(t_{1}^{0}\right)$ with $\delta_{1}$. The resulting lower bound can be lower bounded once more if we substitute $\Psi_{5}$ with one of its upper bounds, and the exponent of $\Psi_{5}$ with one of its lower bounds. This is due to the fact that $\frac{1}{1+A_{1}}>\delta_{1}$ (see CON16 in Table III), and $\Psi_{5}<1$. Note that $\Psi_{5}<1-\delta_{2} A_{2}-\delta_{2} O_{\max }^{-1}$, since $x_{M+1}\left(t_{1}^{0}\right)=0$. Also, note that $\left(1+A_{1}\right) \epsilon_{2}\left[\left(1+A_{2} O_{M+1}\right) \epsilon_{1}\right]^{-1}>\left(1+A_{1}\right) \epsilon_{2}[(1+$ $\left.\left.A_{2} O_{\max }\right) \epsilon_{1}\right]^{-1}$. The above discussion proves that $p_{2}$ of Table $\mathrm{V}$ is indeed a lower bound of $x_{1}\left(t_{2}^{1}\right)$.

\section{APPENDIX E}

In this appendix we prove the validity of ART1 design constraint \#3 in the second competition cycle. In other words, we prove that node $v_{M+2}$ is the first node to be activated after time instance $t_{1}^{1}$. Consider the activity of node $v_{M+2}$ and the activity of a node $v_{j}(j \neq M+1, M+2)$ after time instance $t_{1}^{1}$. The activity of both nodes, after time instance $t_{1}^{1}$, is described by equation (A.3) with $t_{a}=t_{1}^{1}$ and $T_{j}=O_{j}$. If $x_{M+2}\left(t_{1}^{1}\right) \geq$ $x_{j}\left(t_{1}^{1}\right)$, then node $v_{M+2}$ will become supraliminally active before node $v_{j}$ (note that $O_{M+2}>O_{j}$ for $j \neq M+1, M+2$ ). Let us concentrate therefore on the more interesting case where $x_{M+2}\left(t_{1}^{1}\right)<x_{j}\left(t_{1}^{1}\right)$, and in particular on the extreme case where $x_{j}\left(t_{1}^{1}\right)-x_{M+2}\left(t_{1}^{1}\right)$ is substituted by one of its upper bounds. This extreme case is derived below.

Assume that node $v_{M+2}$ receives a bottom-up input $T_{M+2}=$ 0 in the time interval $\left(t_{4}^{1}, t_{1}^{1}\right)$. Hence, the activity of node $v_{M+2}$ in this interval satisfies (A.3) with $x_{j}\left(t_{a}\right)=x_{M+2}\left(t_{4}^{1}\right) \approx$ $-O_{\max }, T_{j}=T_{M+2}=0$, and $t-t_{a}=t_{1}^{1}-t_{4}^{1}$, where $t_{1}^{1}-t_{4}^{1}$ is given by (26). As a result, $x_{M+2}\left(t_{1}^{1}\right)=-O_{\max } \Psi_{1}^{\frac{\epsilon_{1}}{\left(1+A_{1}\right) \epsilon_{2}}}$, where $\Psi_{1}$ was defined in (28). We also assume that node $v_{j}$ $(j \neq M+1, M+2)$ receives a bottom-up input $T_{j}=O_{j}$ in the intervals $\left(t_{4}^{1}, t_{1}^{1}\right)$. Hence, the activity of $v_{j}$ satisfies (A.3) with $x_{j}\left(t_{a}\right) \approx-O_{\max }, T_{j}=O_{j}$, and $t-t_{a}=t_{1}^{1}-t_{4}^{1}$, where $t_{1}^{1}-t_{4}^{1}$ is given by (26). Consequently,

$$
\begin{aligned}
x_{j}\left(t_{1}^{1}\right)= & -O_{\max } \Psi_{1}^{\frac{\left(1+A_{2} O_{j}\right) \epsilon_{1}}{\left(1+A_{1}\right) \epsilon_{2}}}+\frac{O_{j}}{1+A_{2} O_{j}} \\
& \times\left\{1-\Psi_{1}^{\frac{\left(1+A_{2} O_{j}\right) \epsilon_{1}}{\left(1+A_{1}\right) \epsilon_{2}}}\right\} .
\end{aligned}
$$

An upper bound on $x_{j}\left(t_{1}^{1}\right)$ can be found if the $O_{j}$ 's in (E.1) are substituted with $O_{\max }$, and $\Psi_{1}$ is substituted with its lower bound of $p_{6}$ given in Table $\mathrm{V}$. Note that $p_{4}$ in Table $\mathrm{V}$ is a lower bound on $x_{1}\left(t_{4}^{1}\right)$. Also, $-O_{\max }$ is a lower bound on $x_{M+2}\left(t_{1}^{1}\right)$. As a result, we can write that $x_{j}\left(t_{1}^{1}\right)<-p_{5} O_{\max }$, and $x_{M+2}\left(t_{1}^{1}\right)>-O_{\max }$, where $p_{5}$ is defined in Table V. Based on these inequalities, an upper bound on $x_{j}\left(t_{1}^{1}\right)-x_{M+2}\left(t_{1}^{1}\right)$ is given by the term $\left(1-p_{5}\right) O_{\max }$, and this upper bound corresponds to the extreme case that we plan to consider. For this extreme case we will now determine conditions on the values of the bottom-up inputs $O_{j}(j \neq M+1)$ so that node $v_{M+2}$ will be activated before any other node $v_{j}(j \neq M+1, M+2)$. Suppose first that node $v_{M+2}$ becomes supraliminally active before any other node $v_{j}$ $(j \neq M+2)$ in the first layer of the $F_{2}$ field. This event will happen at time $t_{1}^{1}+\Delta t_{M+2}$, where $\Delta t_{M+2}$ is given by the following equation:

$$
\begin{aligned}
\Delta t_{M+2}= & \ln \left[\frac{O_{M+2}-x_{M+2}\left(t_{1}^{1}\right)\left(1+A_{2} O_{M+2}\right)}{O_{M+2}-\delta_{2}\left(1+A_{2} O_{M+2}\right)}\right] \\
& \times \frac{\epsilon_{2}}{1+A_{2} O_{M+2}} .
\end{aligned}
$$

Suppose now that node $v_{j}(j \neq M+1, M+2)$ becomes supraliminally active before any other node in the first layer of the $F_{2}$ field. This event will happen at time $t_{1}^{1}+\Delta t_{j}$, where $\Delta t_{j}$ is given by the following equation:

$$
\Delta t_{j}=\ln \left[\frac{O_{j}-x_{j}\left(t_{1}^{1}\right)\left(1+A_{2} O_{j}\right)}{O_{j}-\delta_{2}\left(1+A_{2} O_{j}\right)}\right] \frac{\epsilon_{2}}{1+A_{2} O_{j}} .
$$

The equations for $\Delta t_{M+2}$ and $\Delta t_{j}$ were derived using equation (A.3). In order to prove the validity of ART1 design constraint \#3 during the second competition cycle, we have to show that under certain AART1-NN parameter constraints, $\Delta t_{M+2}<\Delta t_{j}$. After substituting $x_{M+2}\left(t_{1}^{1}\right)$ with $-O_{\max }$, and $x_{j}\left(t_{1}^{1}\right)$ with $-p_{5} O_{\max }$ in (E.2) and (E.3), respectively, we arrive at

$$
\Delta t_{M+2}=\ln \left[\frac{y_{M+2}+O_{\max }}{y_{M+2}-\delta_{2}}\right] \frac{\epsilon_{2}}{1+A_{2} O_{M+2}}
$$

and

$$
\Delta t_{j}=\ln \left[\frac{y_{j}+p_{5} O_{\max }}{y_{j}-\delta_{2}}\right] \frac{\epsilon_{2}}{1+A_{2} O_{j}}
$$

where $y_{M+2}=\frac{O_{M+2}}{1+A_{2} O_{M+2}}$ and $y_{j}=\frac{O_{j}}{1+A_{2} O_{j}}$. Based on the above equations, it is easy to see that a sufficient condition for $\Delta t_{M+2}$ to be smaller than $\Delta t_{j}$, for $j \neq M+1, M+2$, is the following:

$$
\frac{y_{M+2}+O_{\max }}{y_{M+2}-\delta_{2}}<\frac{y_{j}+p_{5} O_{\max }}{y_{j}-\delta_{2}}
$$


Multiplying both sides of the above inequality with $\left(y_{M+2}-\right.$ $\left.\delta_{2}\right)\left(y_{j}-\delta_{2}\right)$, yields an equivalent inequality:

$$
\begin{aligned}
& -\delta_{2} y_{M+2}+y_{j} O_{\max }-\delta_{2} O_{\max } \\
& \quad<-\delta_{2} y_{j}+p_{5} y_{M+2} O_{\max }-p_{5} \delta_{2} O_{\max }
\end{aligned}
$$

To satisfy this inequality it suffices to guarantee that $y_{j}<$ $p_{5} y_{M+2}$. This is because $-\delta_{2} y_{M+2}-\delta_{2} O_{\max }<-\delta_{2} y_{j}-$ $p_{5} \delta_{2} O_{\max }$. Substituting $y_{j}$ and $y_{M+2}$ with their equals of $O_{j}\left(1+A_{2} O_{j}\right)^{-1}$, and of $O_{M+2}\left(1+A_{2} O_{M+2}\right)^{-1}$, we arrive at the condition

$$
p_{5} O_{j}^{-1}-O_{M+2}^{-1}>\left(1-p_{5}\right) A_{2}
$$

A sufficient condition for the satisfaction of this inequality is constraint CON14 of Table III. Consequently, we have proven that ART1 design constraint \#3 is valid in the second competition cycle, provided that CON14 is satisfied.

\section{APPENDIX F}

In this appendix we will prove that under certain AART1NN design constraints, the reset node becomes subliminally active by time instance $t_{2}^{2}$, and as a result ART1 design constraint \#4 is satisfied in the second competition cycle. Note that at time instance $t_{2}^{2}$, there is no mismatch between bottom-up and top-down inputs at the $F_{1}$ field.

Assume we are at time instance $t_{1}^{1}$, and that the reset node is deactivated at some point prior to time instance $t_{2}^{2}$. We will develop appropriate AART1-NN constraints that prove the correctness of the latter assumption. After time instance $t_{1}^{1}$ the activity of the reset node satisfies (A.7) with $t_{a}=t_{1}^{1}$. Based on (A.7) we can show that the reset node is deactivated at time $t_{1}^{1}+\Delta t_{r 1}$, where $\Delta t_{r 1}=\ln \left[x_{r}\left(t_{1}^{1}\right) \delta_{r}{ }^{-1}\right] A_{r}^{-1} \epsilon_{r}$. If we can demonstrate that under certain AART1-NN parameter constraints, the activity of every node $v_{j}(j \neq M+1)$ at time $t_{1}^{1}+\Delta t_{r 1}$ is below $\delta_{2}$, then we have proven ART1 design constraint \#4. The activity of a node $v_{j}(j \neq M+1)$ after $t_{1}^{1}$ satisfies (A.3) with $t_{a}=t_{1}^{1}$ and $T_{j}=O_{j}$. Hence, at time $t_{1}^{1}+\Delta t_{r 1}$ we can state that

$$
\begin{aligned}
x_{j}\left(t_{1}^{1}+\Delta t_{r 1}\right)= & x_{j}\left(t_{1}^{1}\right) \Psi_{6} \frac{\left(1+A_{2} O_{j}\right) \epsilon_{r}}{A_{r} \epsilon_{2}} \\
& +\frac{O_{j}}{1+A_{2} O_{j}}\left\{1-\Psi_{6} \frac{\left(1+A_{2} O_{j}\right) \epsilon_{r}}{A_{r} \epsilon_{2}}\right\}
\end{aligned}
$$

where $\Psi_{6}=\frac{\delta_{r}}{x_{r}\left(t_{1}^{1}\right)}$. We have already established that an upper bound for the term $x_{j}\left(t_{1}^{1}\right)$ in expression (F.1) is equal to $-p_{5} O_{\max }$ (see Appendix E). Hence, we can write

$$
\begin{aligned}
x_{j}\left(t_{1}^{1}+\Delta t_{r 1}\right)< & -p_{5} O_{\max } \Psi_{6} \frac{\left(1+A_{2} O_{j}\right) \epsilon_{r}}{A_{r} \epsilon_{2}} \\
& +\frac{O_{j}}{1+A_{2} O_{j}}\left\{1-\Psi_{6} \frac{\left(1+A_{2} O_{j}\right) \epsilon_{r}}{A_{r} \epsilon_{2}}\right\} .
\end{aligned}
$$

An upper bound for the right hand side of inequality (F.2) can be found if we substitute $\Psi_{6}$ with one of its lower bounds, and the $O_{j}$ 's with $O_{\max }$. A lower bound on $\Psi_{6}$ is equal to $\delta_{r}$. As a result, $x_{j}\left(t_{1}^{1}+\Delta t_{r 1}\right)<-p_{5} O_{\max } p_{8}+(1-$ $\left.p_{8}\right) O_{\max }\left(1+A_{2} O_{\max }\right)^{-1}$, where $p_{5}$ and $p_{8}$ are defined in Table V. Obviously, ART1 design constraint \#4 is satisfied in the second competition cycle if the AART1-NN parameters are chosen according to CON15 in Table III.

\section{ACKNOWLEDGMENT}

The authors would like to thank the members of the BSP Group for their helpful discussions and suggestions.

\section{REFERENCES}

[1] G. A. Carpenter and S. Grossberg, "A massively parallel architecture for a self-organizing neural pattern recognition machine," Computer Vision, Graphics, and Image Processing , vol. 37, pp. 5 4-115, 1987.

[2] M. W. Hirsch, "Convergent activation dynamics in continuous time networks," Neural Net., vol. 2, no. 5 pp. 331-349, 1989.

[3] S. N. Rasband, Chaotic Dynamics of Nonlinear Systems. New York: Wiley, 1990

[4] D. Ruelle, Elements of Differentiable Dynamics and Bifurcation Theory. San Diego, CA: Academic Press, 1989.

[5] B. Kosko, Neural Networks and Fuzzy Systems: A Dynamical Systems Approach to Machine Intelligence. Englewood Cliffs, NJ: Prentice Hall, 1992.

[6] M. W. Hirsch and S. Smale, Differential Equations, Dynamical Systems, and Linear Algebra. San Diego, CA: Academic Press, 1974.

[7] C. Abdallah, G. L. Heileman, J. W. Howse, IV, and M. Georgiopoulos, "Dynamical neural networks as gradient systems," Tech. Rep. EECE, Univ. New Mexico, pp. 92-100, Sept. 1992.

[8] J. J. Liou, C. S. Ho, M. Georgiopooulos, G. L. Heileman, and C. Christodoulou, "Design and simulation of adaptive resonance theory (ART) neural networks," in SPIE Conf. Proc., Orlando, FL, 1993.

[9] C. S. Ho, J. J. Liou, M. Georgiopoulos, G. L. Heileman, and C. Christodoulou, "Analog circuit design and implementation of an adaptive resonance theory (ART) neural network architecture," Int. J. Elect., vol. 76, no. 2, pp. 271-291, 1994.

[10] F. J. Pineda, "Dynamics and architecture for neural computation," $J$. Complexity, vol. 4, pp. 216-245, 1988.

[11] R. J. Williams and D. Zipser, "A learning algorithm for continually running fully recurrent neural networks," Neural Computation, vol. 1 no. 2, pp. 270-280, 1989.

[12] K. S. Narendra and K. Parthasarathy, "Gradient methods for the optimization of dynamical systems containing neural networks," IEEE Trans. Neural Net., vol. 2, pp. 252-262, 1991

[13] G. A. Carpenter and S. Grossberg, "ART 2: Self-organization of stable category recognition codes for analog input patterns," Appl. Optics, vol 26, no. 23 , pp. $4919-4930,1987$

[14] _ART 3: Hierarchical search using chemical transmitters in selforganizing pattern recognition architectures," Neural Net., vol. 3, no. 2, pp. 129-152, 1990.

[15] M. Georgiopoulos, G. L. Heileman, and J. Huang, "Properties of learning related to pattern diversity in ART1," Neural Net., vol. 4, no. 6, pp. 751-757, 1991.

[16] , "The N-N-N conjecture in ART1," Neural Net., vol. 5, no. 5, pp. 745-753, 1992.

[17] G. A. Carpenter, S. Grossberg, and D. B. Rosen, "ART 2-A: An adaptive resonance algorithm for rapid category learning and recognition," Neural Net., vol. 4, no. 4, pp. 493-504, 1991.

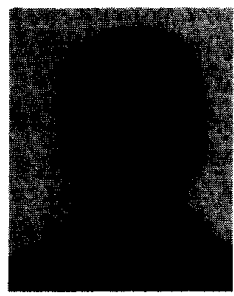

Gregory L. Heileman (S'89-M'89) received the B.S. degree from Wake Forest University in 1982 the M.S. degree in biomedical engineering and mathematics from the University of North CarolinaChapel Hill in 1986, and the Ph.D. degree in computer engineering from the University of Central Florida in 1989

$\mathrm{He}$ is currently an Assistant Professor in the Electrical and Computer Engineering Department at the University of New Mexico, Albuquerque NM. His research interests include neural networks, computational leaming theory, parallel computing, and pattem recognition. Dr. Heileman is a member of the IEEE Computer Society, the Association for Computing Machinery, and the International Neural Network Society. 


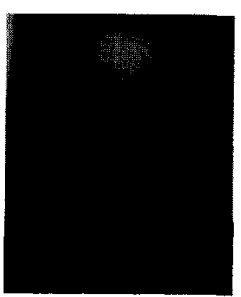

Michael Georgiopoulos (S'84-M'86) received the Diploma in electrical engineering from the National Technical University of Athens, Greece in 1981, and the M.Sc. and Ph.D. degrees in electrical engineering from the University of Connecticut, Storrs, in 1983 and 1986 , respectively.

In January 1987, he joined the University of Central Florida, Orlando, where he is presently and Associate Professor in the Department of Electrical Engineering. His current research interests are in the areas of neural networks and spread spectrum communications.

Dr. Georgiopoulos is a member of the Technical Chamber of Greece and the International Neural Network Society.

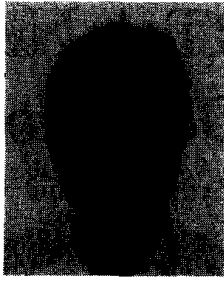

Chaouki Abdallah (S'87-M'88) received the

B.E. degree in electrical engineering in 1981

from Youngstown State University, OH, the M.S. degree in 1982 and the Ph.D. degree in electrical engineering in 1988 from Georgia Tech, Atlanta, GA.

Between 1983 and 1985 he was with SAWTEK Inc., Orlando, FL. In September of 1988 he joined the Department of Electrical And Computer Engineering at the University of New Mexico, Albuquerque, NM where he is presently an Associate Professor. His research interests are in the areas of dynamic neural networks, nonlinear and robust control, and robotics. He is also a co-editor of the book Robot Control: Dynamics, Motion Planning, and Analysis, (IEEE) and co-author of a book titled Robot Control (Macmillan, 1993).

Dr. Abdallah was exhibit chairman of the 1990 International Conference on Acoustics, Speech, and Signal Processing (ICASSP), in Albuquerque, NM. $\mathrm{He}$ is a member of Sigma Xi and Tau Beta Pi. 